\title{
Nonlinear dynamics of core-annular film flows in the presence of surfactant
}

\author{
S. A. KAS-DANOUCHE ${ }^{1}$, D. T. PAPAGEORGIOU U $\mathrm{U}^{2,3}$ AND M. SIEG EL \\ ${ }^{1}$ Departmento de Matemáticas, Universidad de Oriente, Cumaná, Sucre 6101, VENEZUELA \\ ${ }^{2}$ Department of Mathematical Sciences and Center for Applied Mathematics and Statistics, New Jersey Institute of \\ Technology, Newark, New Jersey 07102. USA, \\ ${ }^{3}$ Department of Mathematics, Imperial College London, London SW7 2AZ, U.K.
}

(Received 11 November 2008)

The nonlinear stability of two-phase core-annular flow in a cylindrical pipe is studied. A constant pressure gradient drives the flow of two immiscible liquids of different viscosities and equal densities, and surface tension acts at the interface separating the phases. Insoluble surfactants are included and we assess their effect on the flow stability and ensuing spatio-temporal dynamics. We achieve this by developing an asymptotic analysis in the limit of a thin annular layer - this is usually the relevant regime in applications - to derive a coupled system of nonlinear evolution equations that govern the dynamics of the interface and the local surfactant concentration on it. In the absence of surfactants the system reduces to the Kuramoto-Sivashinsky (KS) equation and its modifications due to viscosity stratification (present when the phases have unequal viscosities) are derived elsewhere. We report on extensive numerical experiments to evaluate the effect of surfactants on KS dynamics (including chaotic states, for example), both in the absence and presence of viscosity stratification. We find that chaos is suppressed in the absence of viscosity differences and that the new flow consists of successive windows (in parameter space) of steady-state travelling waves separated by time-periodic attractors. The intricate structure of the travelling pulses is also explained physically. When viscosity stratification is present we observe a transition from time-periodic dynamics, for instance, to steady-state travelling wave pulses of increasing amplitudes and speeds. Numerical evidence is presented that indicates that the transition occurs through a reverse Feigenbaum cascade in phase space.

\section{Introduction}

The simplest form of a core-annular flow (CAF in the sequel), is a parallel flow consisting of a liquid moving through the tube core of a cylindrical pipe, surrounded by an annular ring of a second immiscible liquid. When an axial pressure gradient is present in a horizontal tube and gravity is absent (or when the tube is vertical in the presence of gravity), there exists an exact parallel flow solution of the Navier-Stokes equations, with the interface separating the two phases circular and concentric with the tube wall. This exact solution is susceptible to instabilities that can produce complex spatio-temporal dynamics of the interface such as interfacial turbulence at zero Reynolds numbers, in addition to topological transitions leading to other flow regimes such as slug or emulsion flows (see Joseph \& Renardy 1993). Besides their fundamental physical and mathematical interest, core-annular flows arise in a host of technological applications including lubricated pipelining (Joseph \& Renardy 1993), liquid-liquid displacements in the presence of a wall wetting layer in porous media (Park \& Homsy 1984), secondary oil recovery (Slattery 1994), and fluid dynamics in the lung (Otis, Johnson, Pedley \& Kamm 1993).

The flow stability is determined by a competition between capillary forces and effects arising from viscosity and density differences (the latter are excluded in the present study). The familiar scenario of the destabilization of cylindrical threads to long waves by the circumferential contribution of the capillary forces, has been established in non-flowing viscous threads surrounded by a second immiscible liquid (Tomotika 1935), and a stationary annular film on the outside surface of a cylinder (Goren 1962). When flow is present the situation is more involved. The paradigm example is that of the stability of Couette-Poiseuille two-phase flow between parallel plates first analyzed by Yih (1967). Yih performed a long-wave stability analysis and 
found that instability is possible if the two fluids have different viscosities. Furthermore, it was found that for thin layers, the flow is stable if the less viscous liquid occupies the thinner layer, and unstable in the reverse regime. It is worth emphasizing that the instability is present at any Reynolds number, however small, and disappears as the Reynolds number vanishes, that is, flow is a necessary condition for its manifestation in contrast to capillary instability. In addition, an interfacial deflection is necessary - planar interfaces are stable - physically, a deflection introduces a velocity perturbation due to the differences in viscosity of the two phases and these perturbations drive the flow in their respective regions (see section 3 also). For a mechanism of the instability the reader is referred to Charru \& Hinch (2000).

In CAF arrangements, it was shown by Hickox (1971) using a long-wave theory as in Yih (1967), that instability to axisymmetric and nonaxisymmetric disturbances arises even in the absence of capillarity (but in the presence of flow) when the annnular fluid is more viscous. A more complete numerical study based on the Orr-Sommerfeld equation was performed by Preziosi, Chen \& Joseph (1989). The main finding is the existence of a range of Reynolds numbers for which the flow is stable to all wavenumbers, that is the viscosity differences act to completely suppress the capillary instability. Such findings were also established in the study of Georgiou, Maldarelli, Papageorgiou \& Rumschitzki (1992) who considered the physically relevant limit of thin annuli and performed an asymptotic analysis in the presence of surface tension, viscosity differences and gravity in a vertical tube arrangement. It is interesting to note that density differences provide dispersive effects alone in this limit.

Nonlinear asymptotic theories for thin annuli have been carried out both in the absence and presence of flow. In the former case Hammond (1983) derives and studies a nonlinear evolution equation that contains the competing effects of surface tension and nonlinearity and is capable of deformations of the order of the annular thickness. Thus, the model can predict the rupture of the annular layer but not the the snap-off of the core. The problem was addressed numerically in the Stokes regime by Newhouse \& Pozrikidis (1992) who verify Hammond's predictions and also present solutions with snap-off of the core for sufficiently thick annuli. The effect of insoluble surfactants on such models have been included by Otis et al. $(1990,1993)$ as well as Halpern \& Grotberg (1993), results in decreased growth rates as determined by linear instability. More complete linear stability studies of surfactant effects on CAF arrangements can be found in Kwak \& Pozrikidis (2001) for quiescent fluids and Kwak, Fyrillas \& Pozrikidis (2001) for liquid threads in extensional flow. The linear stability studies of surfactant-laden liquid threads by Hansen, Peters \& Meijer (1999) and Timmermans \& Lister (2002) are also of interest as well as the nonlinear models and calculations of Craster, Matar \& Papageorgiou (2002).

In the presence of flow the stability of CAFs is more involved. In general, consistent asymptotic theories for thin annuli can be developed when the interfacial amplitude is in the weakly nonlinear regime (an exception is Kerchman 1995 who allows disturbances scaled on the annulus but requires asymptotically small shear in the film). In the absence of viscosity differences Frenkel, Babchin, Levich, Shlang \& Sivashinsky (1987) derive a Kuramoto-Sivashinsky equation for the spatio-temporal evolution and argue that capillary instability is saturated nonlinearly. Papageorgiou, Maldarelli \& Rumschitzki (1990) have shown that when viscosity differences are present (as in lubricated pipelining for example), there is a coupling between the film and core dynamics with the solutions of the Stokes or linearised Navier-Stokes equations in the core required to close the problem. The evolution is governed by a generalized KS equation which contains a linear nonlocal pseudo-differential operator which can be purely dispersive (Stokes limit) or provide both dispersion and growth of wavy disturbances. Papageorgiou et al. (1990) carry out numerical experiments that show that in general the viscosity difference acts to regularize the interfacial dynamics into trains of travelling wave pulses - there are regimes where more complex behavior is seen, however. More detailed computational studies of the KS equation with emphasis placed on transitions to chaotic interfacial dynamics can be found in Papageorgiou \& Smyrlis (1991), Smyrlis \& Papageorgiou (1991, 1996). A more detailed study of the nonlocal equation and a numerical comparison of its solutions with a local model that retains the first two terms in a long-wave expansion of the pseudo-differential operator, has been carried out by Smyrlis \& Papageorgiou (1998), who show that the differences in identifiable attractors such as travelling or time-periodic travelling wave regimes, are almost unidentifiable. Smyrlis \& Papageorgiou(1998)

The problem of CAFs in the presence of flow and surfactants has not received much attention. Of relevance to the present work is the study of Wei \& Rumschitzki (2005) and Blyth, Luo \& Pozrikidis (2006). The former work presents a detailed linear stability study based on the asymptotic expansion of solutions in the limit of 
thin annuli. The stability is reduced to that of a coupled constant coefficient linear system for the interfacial and surfactant perturbations, in the limit when the core dynamics decouple from those of the film. Extensive results are presented that emphasize important differences between the clean and surfactant-laden cases. Similar results are found by Blyth et al. (2006) who note that the presence of surfactant in CAFs does not initiate any additional unstable modes compared to the clean case, in contrast to such phenomena in two-dimensional plane channel flows as studied by Blyth \& Pozrikidis (2004), Frenkel \& Halpern (2002), and Halpern \& Frenkel (2003) (note that the first of these studies also carries out direct numerical simulations in the Stokes limit). The study of Blyth et al. (2006) also follows the instability into the nonlinear regime by use of the immersed boundary method for the Navier-Stokes equations. The computations presented there are not in the thin annulus regime - two sets are presented, having a ratio of undisturbed core radius to tube radius of 0.75 and 0.25 , respectively. In the former case numerical evidence is shown with the interface evolving to multi-valued configurations, while in the latter case (the core is now relatively thin), the evolution tends to core rupture together with thin thread regions in the vicinity of the rupture points in situations when the core is less viscous than the film (a linearly unstable configuration according to the analysis of Hickox 1971.

The results of Blyth et al. (2006) exhibit complicated dynamical scenarios and the parameter space is too large for direct simulations to achieve the detail afforded by model equations as in the present work. We consider thin annuli and carry out an asymptotic analysis that is structured so as to retain nonlinearity in the leading order evolution. The technical difficulty arises in the coupling between the interfacial and surfactant perturbation evolutions; this necessitates the identification of canonical asymptotic regimes that allow nonlinearity to enter in a Galilean frame fixed to the unperturbed interfacial velocity. This is apparently straight forward in the clean case but in the presence of surfactants the initial surfactant concentration needs to be chosen accordingly in order to retain nonlinearity (any other choice of initial surfactant distribution merely reproduces the linear system of Wei \& Rumschitzki 2005). Our analysis produces canonical evolution equations valid for a wide range of parameters. The resulting system couples the generalized KS equation of Papageorgiou et al. (1990) to a convection-diffusion equation for the surfactant and all our numerical evidence indicates that the system most likely possesses global existence of solutions. A proof of this is still missing.

The structure of the rest of the paper is as follows. Section 2 presents the fully nonlinear non-dimensional problem and introduces the various dimensionless groups. Section 3 is devoted to the asymptotic derivation of the evolution equations and a careful explanation of the scalings involved in producing nonlinear dynamics. Section 4 gives a canonical rescaling for the system along with linear stability properties, while section 5 describes our numerical methods to solve the system as a periodic initial value problem. Section 6 contains our results for an exhaustive range of parameters. We close with our conclusions in section 7 .

\section{Mathematical model and governing equations}

Our problem consists of an annular liquid film (fluid 2), surrounding an infinitely long cylindrical fluid core (fluid 1). Fluid 1 has viscosity $\mu_{1}$ and occupies the core region $0 \leq r<S(z, t)$, while fluid 2 has viscosity $\mu_{2}$ and occupies the annular region $S(z, t)<r<R_{2}$, where $R_{2}$ is the tube radius and $S(z, t)$ is the interface. The fluid densities are assumed to be equal and are denoted by $\rho$, and the undisturbed state is given by $S(z, t)=R_{1}$. Throughout this work we assume that the flows and geometry are axisymmetric. For horizontal tubes, this is a reasonable approximation when the Bond number $B_{0}=\frac{\rho g R_{2}^{2}}{\sigma} \ll 1$, and this is assumed here (see Hammond 1983). In the case of vertical tubes, gravitational effects can be easily incorporated into the present analysis, but we do not pursue this here.

We introduce cylindrical polar coordinates $\mathbf{x}=(r, \theta, z)$ with associated velocity components $\mathbf{u}_{1}=$ $\left(u_{1}, 0, w_{1}\right)$ for the fluid core and $\mathbf{u}_{2}=\left(u_{2}, 0, w_{2}\right)$ for the fluid film and denote pressures in the core and film region by $p_{1}$ and $p_{2}$, respectively. The flow in regions 1 and 2 are governed by the incompressible Navier-Stokes equations

$$
\begin{aligned}
\rho\left(u_{i t}+u_{i} u_{i r}+w_{i} u_{i z}\right) & =-p_{i r}+\mu_{i}\left(\nabla^{2} u_{i}-\frac{u_{i}}{r^{2}}\right) \\
\rho\left(w_{i t}+u_{i} w_{i r}+w_{i} w_{i z}\right) & =-p_{i z}+\mu_{i} \nabla^{2} w_{i}
\end{aligned}
$$




$$
\frac{1}{r}\left(r u_{i}\right)_{r}+w_{i z}=0
$$

where subscripts $i=1,2$ refer to the particular flow region, and letter subscripts $t, r, z$ denote partial derivatives. The Laplacian in cylindrical coordinates is $\nabla^{2} \equiv \frac{1}{r} \frac{\partial}{\partial r}\left(r \frac{\partial}{\partial r}\right)+\frac{\partial^{2}}{\partial z^{2}}$.

The boundary conditions are those of no-slip at the wall and continuity of velocities at the interface. In addition we impose the usual kinematic condition at the interface. In terms of the aforementioned variables, these boundary conditions are

$$
\begin{array}{r}
u_{2}\left(R_{2}, z, t\right)=w_{2}\left(R_{2}, z, t\right)=0, \\
u_{1}(S, z, t)=u_{2}(S, z, t), \quad w_{1}(S, z, t)=w_{2}(S, z, t), \\
u_{i}(S, z, t)=S_{t}+w_{i}(S, z, t) S_{z} .
\end{array}
$$

The normal and tangential balances are given by

$$
\begin{gathered}
{\left[-p_{i}+\frac{2 \mu_{i}}{1+\left(S^{\prime}\right)^{2}}\left(\left(S^{\prime}\right)^{2} \frac{\partial w_{i}}{\partial z}-S^{\prime}\left(\frac{\partial u_{i}}{\partial z}+\frac{\partial w_{i}}{\partial r}\right)+\frac{\partial u_{i}}{\partial r}\right)\right]_{1}^{2}=\frac{\sigma(\Gamma)}{S \sqrt{1+\left(S^{\prime}\right)^{2}}}\left\{1-\frac{S S^{\prime \prime}}{1+\left(S^{\prime}\right)^{2}}\right\}} \\
{\left[\frac{\mu_{i}}{1+\left(S^{\prime}\right)^{2}}\left(2 S^{\prime}\left(\frac{\partial u}{\partial r}-\frac{\partial w}{\partial z}\right)+\left(1-\left(S^{\prime}\right)^{2}\right)\left(\frac{\partial u}{\partial z}+\frac{\partial w}{\partial r}\right)\right)\right]_{1}^{2}=\frac{-1}{\sqrt{1+\left(S^{\prime}\right)^{2}}} \frac{\partial \sigma}{\partial z}}
\end{gathered}
$$

where we have employed the notation $\left[f_{i}\right]_{1}^{2}=f_{2}-f_{1}$. The last term in (2.8) represents the tangential stress (Marangoni force) which results from the dependence of the interfacial tension $\sigma$ on the non-uniform surfactant concentration $\Gamma(z, t)$. The presence of surfactant generally acts to lower interfacial tension, owing to the repulsion of the polar surfactant molecules. The precise nature of the dependence of surface tension on $\Gamma$ is given by an equation of state of the form $\sigma=\sigma(\Gamma)$. If the surfactant is present in dilute concentration, a linear relation between $\sigma$ and $\Gamma$ may be assumed, i.e.

$$
\sigma=\sigma_{0}\left(1-\beta \Gamma / \Gamma_{\infty}\right)
$$

where $\Gamma_{\infty}$ is the maximum packing concentration of surfactant and $\beta=\frac{R T \Gamma_{\infty}}{\sigma_{0}}$, where $R$ is the gas constant and $T$ is the absolute temperature. Note that $\beta$ determines the sensitivity of interfacial tension to changes in surfactant concentration.

A convection-diffusion equation for surfactant transport is derived in Wong, Rumschitzki, and Maldarelli (1996) ; in Cartesian coordinates this equation takes the form

$$
\frac{\partial \Gamma}{\partial t}-\frac{\partial \mathbf{x}}{\partial t} \cdot \nabla_{s} \Gamma+\nabla_{s}\left(\Gamma w_{s}\right)-D_{s} \nabla_{s}^{2} \Gamma+\Gamma \kappa \mathbf{u} \cdot \mathbf{n}=\mathbf{0},
$$

where $\mathbf{x}$ is the position vector of the interface, $w_{s}$ is the surface velocity at the interface, $D_{s}$ is the constant interfacial surfactant diffusivity, $\kappa$ is the curvature of the interface taken positive for convex surface shapes, and $\mathbf{n}$ is the unit normal vector directed outward from the core region 1 . The notation $\nabla_{s}$ refers to the surface gradient operator. Equation (2.10) is easily rewritten in cylindrical-polar coordinates, and takes the form (see Kas-Danouche (2002))

$$
\begin{aligned}
\frac{\partial \Gamma}{\partial t} & -\frac{S_{t} S^{\prime}}{1+\left(S^{\prime}\right)^{2}} \frac{\partial \Gamma}{\partial z}+\frac{1}{S \sqrt{1+\left(S^{\prime}\right)^{2}}}\left\{\frac{\partial}{\partial z}\left[\frac{S \Gamma}{\sqrt{1+\left(S^{\prime}\right)^{2}}}\left(w+S^{\prime} u\right)\right]\right\} \\
& -D_{s} \frac{1}{S \sqrt{1+\left(S^{\prime}\right)^{2}}} \frac{\partial}{\partial z}\left(\frac{S}{\sqrt{1+\left(S^{\prime}\right)^{2}}} \frac{\partial \Gamma}{\partial z}\right)+\frac{\Gamma}{S\left(1+\left(S^{\prime}\right)^{2}\right)}\left[1-\frac{S S^{\prime \prime}}{1+\left(S^{\prime}\right)^{2}}\right]\left(-S^{\prime} w+u\right)=0
\end{aligned}
$$

Here, a prime denotes differentiation with respect to $z$.

\subsection{Nondimensionalization}

The preceeding problem can be recast in terms of nondimensional quantities if lengths are rescaled by the undisturbed core radius $R_{1}$, velocities by the baseflow centerline velocity $W_{0}$, time by $R_{1} / W_{0}$, interfacial tension by the clean surface tension $\sigma_{0}$, and pressure by $\rho W_{0}^{2}$. The resulting equations are now listed using 
the same notation for nondimensional as for dimensional variables (the dimensionless groups that appear are defined in equation (2.20).

The dimensionless Navier-Stokes Equations and no-slip conditions are

$$
\begin{aligned}
\left(u_{i}\right)_{t}+u_{i}\left(u_{i}\right)_{r}+w_{i}\left(u_{i}\right)_{z} & =-\left(p_{i}\right)_{r}+\frac{1}{R e_{i}}\left[\nabla^{2} u_{i}-\frac{u_{i}}{r^{2}}\right], \\
\left(w_{i}\right)_{t}+u_{i}\left(w_{i}\right)_{r}+w_{i}\left(w_{i}\right)_{z} & =-\left(p_{i}\right)_{z}+\frac{1}{R e_{i}} \nabla^{2} w_{i}, \\
\left(u_{i}\right)_{r}+\frac{1}{r} u_{i}+\left(w_{i}\right)_{z} & =0,
\end{aligned}
$$

where $i=1,2$ for core and film respectively, and

$$
\begin{gathered}
u_{2}=w_{2}=0 \quad \text { at } \quad r=\frac{R_{2}}{R_{1}}, \\
u_{1}=u_{2}, w_{1}=w_{2} \quad \text { on } \quad r=S(z, t) .
\end{gathered}
$$

The surfactant transport equation takes the form

$$
\begin{aligned}
\frac{\partial \Gamma}{\partial t} & -\frac{S_{t} S^{\prime}}{1+\left(S^{\prime}\right)^{2}} \frac{\partial \Gamma}{\partial z}+\frac{1}{S \sqrt{1+\left(S^{\prime}\right)^{2}}}\left\{\frac{\partial}{\partial z}\left[\frac{S \Gamma}{\sqrt{1+\left(S^{\prime}\right)^{2}}}\left(w+S^{\prime} u\right)\right]\right\} \\
& -\frac{1}{P_{e}} \frac{1}{S \sqrt{1+\left(S^{\prime}\right)^{2}}} \frac{\partial}{\partial z}\left(\frac{S}{\sqrt{1+\left(S^{\prime}\right)^{2}}} \frac{\partial \Gamma}{\partial z}\right)+\frac{\Gamma}{S\left(1+\left(S^{\prime}\right)^{2}\right)}\left[1-\frac{S S^{\prime \prime}}{1+\left(S^{\prime}\right)^{2}}\right]\left(-S^{\prime} w+u\right)=0
\end{aligned}
$$

The normal stress balance is given by

$$
\left[p\left(1+\left(S^{\prime}\right)^{2}\right)-\frac{2}{R e_{i}}\left[\left(S^{\prime}\right)^{2} w_{z}-S^{\prime}\left(u_{z}+w_{r}\right)+u_{r}\right]\right]_{1}^{2}=\frac{J(1-\beta \Gamma)}{R e_{1}^{2}}\left\{S^{\prime \prime}-\frac{1}{S}\left[1+\left(S^{\prime}\right)^{2}\right]\right\}\left[1+\left(S^{\prime}\right)^{2}\right]^{-\frac{1}{2}} 2
$$

while the tangential stress balance becomes

$$
\left[m_{i}\left[2 S^{\prime}\left(u_{r}-w_{z}\right)+\left[1-\left(S^{\prime}\right)^{2}\right]\left(u_{z}+w_{r}\right)\right]\right]_{1}^{2}=\frac{\beta \Gamma_{z}}{C_{a}}\left[1+\left(S^{\prime}\right)^{2}\right]^{\frac{1}{2}},
$$

where $m_{1}=1$ and $m_{2}=m$.

Finally, the kinematic condition reads

$$
u=\frac{\partial S}{\partial t}+w \frac{\partial S}{\partial z}=S_{t}+w S^{\prime}
$$

Note that equations (2.16)-(2.19) are evaluated at the interface $r=S(z, t)$.

The nondimensional groups appearing above are defined by

$$
R e_{i}=\frac{\rho W_{0} R_{1}}{\mu_{i}}, \quad P e=\frac{W_{0} R_{1}}{D_{s}}, \quad a=\frac{R_{2}}{R_{1}}, \quad J=\frac{\rho \sigma_{0} R_{1}}{\mu_{1}^{2}}, \quad m=\frac{\mu_{2}}{\mu_{1}}, \quad C a=\frac{\mu_{1} W_{0}}{\sigma_{0}}, \quad M_{a}=\frac{\beta}{C_{a}},
$$

where

$$
W_{0}=\frac{F}{4 \mu_{1} \mu_{2}}\left[\left(\mu_{2}-\mu_{1}\right) R_{1}^{2}+\mu_{1} R_{2}^{2}\right],
$$

is the dimensionless centerline velocity and $\nabla p=-F \hat{\mathbf{z}}, F>0$, in the undisturbed state. We introduce the parameter $\epsilon>0$ defined by

$$
\epsilon=a-1
$$

where $a$ is the ratio of tube to undisturbed core radius given in (2.20); $\epsilon$ is a measure of the thickness of the annulus and the thin annular limit is obtained when $\epsilon \ll 1$. The parameter $M_{a}$ is the Marangoni number which plays an important role in applications - see Wei \& Rumschitzki (2005).

When the driving force is a constant pressure gradient, it is easily seen that an exact steady solution to 
$(2.12-2.19)$ is $S=1, \Gamma=\Gamma_{0}, \bar{p}_{z}=-\frac{F R_{1}}{\rho W_{0}^{2}}$, and velocities and pressures are given by

$$
\begin{aligned}
\bar{w}_{1} & =1-\frac{m r^{2}}{a^{2}+m-1}, \quad 0 \leq r \leq 1, \\
\bar{w}_{2} & =-\frac{r^{2}-a^{2}}{a^{2}+m-1}, \quad 1 \leq r \leq a, \\
\bar{p}_{2}-\bar{p}_{1} & =-\frac{J\left(1-\beta \Gamma_{0}\right)}{R e_{1}^{2}} .
\end{aligned}
$$

Overbars are used to denote the base state velocities and pressures. We express the interface as

$$
S(z, t)=1+\delta_{1}(\epsilon) H(z, t)
$$

with $H$ of order one and $\delta_{1} \ll 1$, and for later reference note that

$$
\left.\bar{w}_{2}\right|_{r=1+\delta_{1} H}=\frac{(2+\varepsilon) \varepsilon}{m+2 \varepsilon+\varepsilon^{2}}-\frac{2 \delta_{1} H+\delta_{1}^{2} H^{2}}{m+2 \varepsilon+\varepsilon^{2}} \equiv \bar{w}_{c}+\bar{w}_{2 H} .
$$

Here $\bar{w}_{c}$ is the constant part of the base state velocity at the interface and $\bar{w}_{2 H}$ is the part induced by the interfacial deformation $H$. Similarly, $\bar{w}_{1}=\bar{w}_{c}+\bar{w}_{1 H}$ where

$$
\left.\bar{w}_{1 H}\right|_{r=1+\delta_{1} H}=-\frac{2 m \delta_{1} H+m \delta_{1}^{2} H^{2}}{m+2 \varepsilon+\varepsilon^{2}} .
$$

The stated nonlinear time-dependent problem must in general be addressed by direct numerical simulations. However, this is not an easy task due to the moving interface and the large number of physical parameters (see the six dimensionless groups in equation (2.20)). In what follows we make analytical progress by exploring possible canonical asymptotic regimes when $\delta_{1}$ and $\varepsilon$ are small numbers. Physically, this corresponds to the thin annulus limit which is of practical interest in applications.

\section{Derivation of the evolution equations}

\subsection{Scalings}

Due to the large number of parameters, it is essential to identify the physical limits which asymptotic analyses may be able to describe. Throughout this work capillarity is important, so that flows are driven to leading order by the surface tension induced pressure jump across the interface. We will also be concerned with canonical limits that incorporate nonlinear effects; asymptotic limits that lead to linear equations are discussed in Wei \& Rumschitzki (2005). Where possible, we will couple the dynamics of the film with the core. Lastly, we will be concerned with the weakly nonlinear evolution of the interface so that the interfacial amplitude is asymptotically smaller than the undisturbed annular layer thickness, i.e. $\delta_{1} \ll \varepsilon$. For the cylindrical shear flow considered here linear growth saturates in this regime (Papageorgiou et al. 1990). The fully nonlinear regime with $\delta_{1} \sim \varepsilon$ can occur in the no-flow case and is considered in Hammond (1983), and Halpern and Grotberg (1993). by

We begin the analysis by assuming the interface position is given by (2.26) and the surfactant concentration

$$
\Gamma(z, t)=\Gamma_{0}+\delta_{2}(\varepsilon) \tilde{\Gamma}(z, t)
$$

with $\delta_{2}$ to be determined. The parameter $\Gamma_{0}$ is small, in view of the assumption of a dilute surfactant concentration - see (2.9).

In the film (Region 2), we introduce a local variable $r=a-\varepsilon y$ (the variable $y$ is zero at the pipe wall and $1-\delta_{1} H(z, t) / \varepsilon$ at the interface). It is convenient to decompose all variables (pressure, velocity, etc.) into a sum of a base state and a perturbed quantity, e.g. $p_{i}=\bar{p}_{i}+\tilde{p}_{i}$ for $i=1,2$ where the overbar denotes the base state and the tilde denotes a perturbation from this state. Re-scaling the continuity equation (2.14) using the new local film variable $y$ and balancing terms for small $\varepsilon$, immediately leads to the scaling

$$
\tilde{u}_{2} \sim \varepsilon \tilde{w}_{2}
$$

in the film, whereas $\tilde{u}_{1} \sim \tilde{w}_{1}$ in the core. The pressures can be estimated using the normal stress balance 
equation (2.17) along with (3.1). This leads to $\bar{p}_{1}-\bar{p}_{2}=J\left(1-\beta \Gamma_{0}\right) / R_{e 1}^{2}$ and

$$
\tilde{p}_{2}-\tilde{p}_{1}+\mathcal{O}\left(\delta_{1} \tilde{w}_{2}\right)=\frac{J\left(1-\beta \Gamma_{0}\right)}{R_{e 1}^{2}} \delta_{1}\left(H+H_{z z}\right)+\frac{J}{R_{e 1}^{2}} \beta \delta_{2} \tilde{\Gamma}+\mathcal{O}\left(\delta_{1}^{2}, \delta_{1} \delta_{2}\right) .
$$

Note that the second term on the right hand side (RHS) involving $\tilde{\Gamma}$ is due to surfactant induced surface tension variations; since $\beta \ll 1$ by assumption, these variations compete with the curvature terms only if $\delta_{1} \sim \beta \delta_{2}$. Either way, we are led to the following magnitudes for the perturbation pressures

$$
\tilde{p}_{1,2} \sim \frac{J \delta_{1}}{R_{e 1}^{2}}=\frac{\delta_{1}}{C_{a} R_{e 1}}
$$

the last equality following from (2.20). Considering the momentum equations (2.12)-(2.13) in Region 2, the leading order balance produces the lubrication approximation $\tilde{p}_{2 y}=0$ and

$$
-\tilde{p}_{2 z}+\frac{1}{\varepsilon^{2} R_{e 2}} \tilde{w}_{2 y y}=0 .
$$

Equations (3.2), (3.4), (3.5) and the fact that $R_{e 1}=m R_{e 2}$ are used to obtain the estimates

$$
\tilde{w}_{2} \sim \frac{\varepsilon^{2} \delta_{1}}{m C_{a}}, \quad \tilde{u}_{2} \sim \frac{\varepsilon^{3} \delta_{1}}{m C_{a}} .
$$

Next, we identify the leading order terms in the tangential stress balance equation (2.18). The perturbation velocities $\tilde{w}_{1}, \tilde{u}_{1}$ in the core enter at leading order, and since $\tilde{w}_{1} \sim \tilde{u}_{1}$, it is sufficient to estimate $\tilde{w}_{1}$. The main contribution comes from the viscosity difference and the shear base flow in the vicinity of the interface. Equations (2.27) and (2.27) imply

$$
\left.\left(\bar{w}_{1}-\bar{w}_{2}\right)\right|_{r=1+\delta_{1} H}=\frac{2(1-m) \delta_{1} H}{m}+\mathcal{O}\left(\delta_{1}^{2}, \delta_{1} \varepsilon\right) .
$$

This velocity jump, which disappears when the viscosities are equal $(m=1)$, fixes the perturbation velocities in the core

$$
\tilde{w}_{1} \sim \tilde{u}_{1} \sim \frac{\delta_{1}}{m}
$$

The velocity scales (3.6) and (3.8) now determine the core perturbation pressure to be $\tilde{p}_{1}=\mathcal{O}\left(\delta_{1} / m\right)$. Considering the tangential stress balance (2.18) we see that the largest film contribution is of order $m \tilde{w}_{2} / \varepsilon$ (on use of (3.6) this becomes $\mathcal{O}\left(\varepsilon \delta_{1} / C_{a}\right)$ ), the largest core contribution is of order $\delta_{1} / m$ and the largest Marangoni contribution on the right hand side is of size $\beta \delta_{2} / C_{a}$. Coupling of film and core dynamics and retention of Marangoni forces implies that all three terms are in balance, leading to

$$
C_{a} \sim m \varepsilon, \quad \delta_{2} \sim \frac{\varepsilon \delta_{1}}{\beta} .
$$

If $C_{a} \ll m \varepsilon$ then there is no coupling between film and core and the film problem closes without the need of the solutions in the core region - this is the case studied by Wei \& Rumschitzki (2005) . In the present analysis we will insist on coupling and recover the decoupled problem as a special case by setting $m=1$. Recall from the discussion just below (3.3) that when surface tension variations are as important as the curvature terms then $\delta_{1} \sim \beta \delta_{2}$. This gives $\Gamma_{z}=0$ to leading order. We do not consider this further here, but see Wei \& Rumschitzki (2005) for some additional comments in the linear regime.

We next consider scalings for the surfactant concentration and kinematic equations (2.16) and (2.19). Evaluating (2.19) at $r=1+\delta_{1} H$ and using the scales (3.6) to define new order one dependent variables $U_{2}$, $W_{2}$ by,

$$
\tilde{u}_{2}=\frac{\varepsilon^{3} \delta_{1}}{m C_{a}} U_{2}, \quad \tilde{w}_{2}=\frac{\varepsilon^{2} \delta_{1}}{m C_{a}} W_{2}
$$

gives

$$
\frac{\varepsilon^{3} \delta_{1}}{m C_{a}} U_{2}=\delta_{1} H_{t}+\frac{2 \varepsilon \delta_{1}}{m+2 \varepsilon} H_{z}-\frac{2 \delta_{1}^{2}}{m+2 \varepsilon} H H_{z}+\frac{\varepsilon^{2} \delta_{1}^{2}}{m C_{a}} W_{2} H_{z}+\text { h.o.t. }
$$

where h.o.t. denotes higher order terms. Equation (3.9) shows that the last term is small compared with the 
left hand side term (unless $\delta_{1} \sim \varepsilon$, corresponding to strongly nonlinear disturbances which must be dealt with by direct simulations). Assuming $m=\mathcal{O}(1)$ the left hand side of (3.11) is small relative to the second term on the right hand side (base flow term) since $\left(\varepsilon^{2} \delta_{1} / m^{2}\right) \ll\left(\varepsilon \delta_{1} / m\right)$. The base flow term can balance the unsteady term by the time-scale change $\partial_{t} \rightarrow \varepsilon \partial_{\tau}$, to yield $H_{\tau}+(2 / m) H_{z}=0$ to leading order. In the surfactant-free case (see Papageorgiou et al. (1990), Georgiou et al. (1992)) a Galilean translation removes this leading order term to produce nonlinear dynamics at the next order. Such a removal is in general not possible due to the coupling with the surfactant concentration equation (2.16). To avoid trivial solutions Wei and Rumschitzki relax the film-core coupling condition (3.9) by allowing a smaller $C_{a}$ in order to make the $U_{2}$ term on the left hand side of (3.11) balance the unsteady and base flow terms; this leads to $C_{a} \sim \varepsilon^{2}$. The analysis inevitably leads to linear dynamics and no film-core coupling, details of which are provided in Wei \& Rumschitzki (2005) . Our objective is to explore in detail scalings that enable nonlinearity to be included in a consistent asymptotic manner, and this is pursued next.

The key in obtaining nonlinear dynamics is to have relatively large $C_{a}$ so that leading order term $H_{t}+$ $(2 \varepsilon / m) H_{z}$ can be removed by a Galilean transformation. Failure to do so leads to a linear response as described in Wei \& Rumschitzki (2005). Consider equation (2.16) at $r=1+\delta_{1} H$ which reads

$$
\begin{aligned}
\delta_{2} \tilde{\Gamma}_{t}+\frac{2 \varepsilon \delta_{2}}{m} \tilde{\Gamma}_{z}-\frac{2 \delta_{1} \Gamma_{0}}{m} H_{z}-\frac{2 \delta_{1} \delta_{2}}{m}(\tilde{\Gamma} H)_{z}+\frac{2 \varepsilon \delta_{1} \Gamma_{0}}{m} H_{z} & -\frac{2 \delta_{1}^{2} \Gamma_{0}}{m}\left(H^{2}\right)_{z}+\frac{\varepsilon^{2} \delta_{1} \Gamma_{0}}{m C_{a}} W_{2 z} \\
& =\frac{\delta_{2}}{P_{e}} \tilde{\Gamma}_{z z}+\text { h.o.t. }
\end{aligned}
$$

In order to obtain a nonlinear response, the term $\delta_{2} \tilde{\Gamma}_{t}+\left(2 \varepsilon \delta_{2} / m\right) \tilde{\Gamma}_{z}$ must dominate to leading order so that it can be removed by the same Galilean transformation that operates on the kinematic condition (3.11). The third term on the left hand side dominates the fifth, sixth and seventh terms (the last result follows on use of $C_{a} \sim m \varepsilon$ with $m=\mathcal{O}(1)$ ). This observation allows us to balance the nonlinear fourth term with the linear third term as long as

$$
\Gamma_{0} \sim \delta_{2} \quad \Rightarrow \quad \Gamma_{0}=\delta_{2} \bar{\Gamma}_{0} \quad\left(\bar{\Gamma}_{0}=\mathcal{O}(1)\right) .
$$

A balance of interfacial diffusion requires

$$
P_{e}=\frac{1}{\delta_{1}} \bar{P}_{e} \gg 1
$$

The appropriate change of time scale that allows unsteadiness in the nonlinear evolution is

$$
\frac{\partial}{\partial t} \rightarrow-\frac{2 \varepsilon}{m} \frac{\partial}{\partial z}+\delta_{1} \frac{\partial}{\partial \tau}
$$

Finally, inserting (3.13)-(3.15) into (3.12) and keeping leading order terms yields the following equation for $\tilde{\Gamma}$ :

$$
\tilde{\Gamma}_{\tau}-\frac{2 \bar{\Gamma}_{0}}{m} H_{z}-\frac{2}{m}(\tilde{\Gamma} H)_{z}=\frac{1}{\bar{P}_{e}} \tilde{\Gamma}_{z z}
$$

Note that $\delta_{2}$ is not yet specified.

It remains to determine $\delta_{1}$ and the evolution of $H$ from the kinematic condition (3.11). Substituting the transformation (3.15) into (3.11) makes the right hand side of (3.11) of order $\delta_{1}^{2}$. Inserting the estimates (3.9) into the left hand side of (3.11) shows that a balance is possible if

$$
\varepsilon^{2} \delta_{1} \sim \delta_{1}^{2} \quad \Rightarrow \quad \delta_{1} \sim \varepsilon^{2}
$$

and the resulting evolution equation is

$$
\left.\frac{1}{m C_{a 0}} U_{2}\right|_{y=1}=H_{\tau}-\frac{2}{m} H H_{z},
$$

where we have written $C_{a}=\varepsilon C_{a 0}$ with $C_{a 0}=\mathcal{O}(1)$ and assumed that $m=\mathcal{O}(1)$. It remains to determine $\left.U_{2}\right|_{y=1}$ in terms of $H$ and $\tilde{\Gamma}$ in order to close the system. Before presenting that calculation, we determine the different parameter regimes that our canonical nonlinear system can describe. We know from (3.9) that Marangoni stresses will be important if $\delta_{2} \sim \varepsilon \delta_{1} / \beta$. In addition, equation (3.13) shows that $\delta_{2}$ is of the same order as the mean initial surfactant concentration $\Gamma_{0}$; combining the two gives $\beta \sim \varepsilon^{3} / \Gamma_{0}$. Using these 
estimates enables us to write the Marangoni number (see (2.20)),

$$
M_{a} \sim \frac{\varepsilon^{2}}{\Gamma_{0}}
$$

It can be seen that flows which are characterized by small, order one, or large Marangoni numbers are covered. Consideration of equations (3.9), (3.13) and (3.19) have identified the following three canonical scalings:

$$
\begin{gathered}
M_{a} \sim 1, \quad \Gamma_{0} \sim \varepsilon^{2}, \quad \beta \sim \varepsilon, \\
\varepsilon^{2} \ll M_{a} \ll 1, \quad \varepsilon^{2} \ll \Gamma_{0} \ll 1, \quad \varepsilon^{3} \ll \beta \ll \varepsilon, \\
1 \ll M_{a} \ll \varepsilon^{-1}, \quad \varepsilon^{3} \ll \Gamma_{0} \ll \varepsilon^{2}, \quad \varepsilon \ll \beta \ll 1 .
\end{gathered}
$$

All the regimes above produce a nonlinear response governed by the same canonical system which is solved numerically in later sections. The initial $\Gamma_{0}$ distribution sets the parameter values and we see that in the dilute limit the Marangoni numbers are large as expected.

\subsection{Asymptotic expansions and the final equations}

The precise form of the asymptotic equations depends on the scale of $R e_{1}$, which is not determined by the previous balance arguments. In the following we choose $R e_{1} \sim \varepsilon$, which provides the simplest situation in which the film and core dynamics couple and the core flow is governed to leading order by the Stokes equations. Other scales are possible, $R e_{1}=\mathcal{O}(1)$ for example, and lead to different evolution equations with linearized Navier-Stokes flow in the core (see Papageorgiou et al. 1990). For completeness we present a brief derivation of the case $R e_{1} \sim \varepsilon$ and refer the reader to Papageorgiou et al. (1990) for details.

Employing the scalings (3.4), (3.6), (3.8), (3.9) and the notation introduced in (3.10) along with (3.17), we express film quantities as $u_{2}=\varepsilon^{4} U_{2}+\mathcal{O}\left(\varepsilon^{5}\right), w_{2}=\bar{w}_{2}+\varepsilon^{3} W_{2}+\mathcal{O}\left(\varepsilon^{4}\right), p_{2}=\bar{p}_{2}+P_{20}+\varepsilon P_{21}+\mathcal{O}\left(\varepsilon^{2}\right)$, while in the core we expand the solutions as $u_{1}=\varepsilon^{2} U_{1}+\mathcal{O}\left(\varepsilon^{3}\right), w_{1}=\bar{w}_{1}+\varepsilon^{2} W_{1}+\mathcal{O}\left(\varepsilon^{3}\right)$ and $p_{1}=\bar{p}_{1}+\varepsilon P_{11}+\mathcal{O}\left(\varepsilon^{2}\right)$. Setting $R_{1}=\varepsilon \lambda$ with $\lambda=\mathcal{O}(1)$ and substituting film variables into (2.12)-(2.14) gives to leading order

$$
P_{20 z}-\frac{m}{\lambda} W_{2 y y}=0, \quad P_{20 y}=0, \quad W_{2 z}=U_{2 y} .
$$

Since $P_{20}$ is independent of $y$, the system (3.23) can be integrated to yield the leading order axial and radial velocities in the film

$$
W_{2}=\frac{\lambda}{m}\left(\frac{1}{2} P_{20 z} y^{2}+A(z, t) y\right), \quad U_{2}=\frac{\lambda}{m}\left(\frac{1}{6} P_{20 z z} y^{3}+\frac{1}{2} A_{z}(z, t) y^{2}\right) .
$$

The function $A(z, \tau)$ is to be determined.

At the interface, the leading order normal and tangential stress balance boundary conditions (equations (2.17) and (2.18)) become

$$
\begin{gathered}
P_{20}=\frac{1}{\lambda C_{a 0}}\left(H+H_{z z}\right), \\
m W_{2 y}(1, z, t)+U_{1 z}(1, z, t)+W_{1 r}(1, z, t)=-\frac{\beta_{0}}{C_{a 0}} \tilde{\Gamma}_{z},
\end{gathered}
$$

where we have used $C_{a}=\varepsilon C_{a 0}$ and $\beta=\left(\varepsilon^{3} / \delta_{2}\right) \beta_{0}$ - see (3.9) and (3.17). The kinematic condition (3.11) combined with a Galilean transformation, yields, to leading order,

$$
U_{2}(1, z, t)=H_{\tau}-\frac{2}{m} H H_{z}
$$

Invoking continuity of axial velocity (using (2.27), (2.27) and the scalings (3.17)) and continuity of radial velocity, lead to the expressions

$$
\left.W_{1}\right|_{r=1}=2 H\left(1-\frac{1}{m}\right),\left.\quad U_{1}\right|_{r=1}=0
$$


The function $A(z, \tau)$ is still unknown and can be determined once the core problem is solved. Using the expressions introduced earlier yields the following leading order Stokes flow in the core,

$$
\begin{aligned}
\lambda P_{11 r} & =\nabla^{2} U_{1}-\frac{U_{1}}{r^{2}}, \\
\lambda P_{11 z} & =\nabla^{2} W_{1}, \\
\frac{1}{r}\left(r U_{1}\right)_{r} & +W_{1 z}=0 .
\end{aligned}
$$

This elliptic problem in a non-slender region causes non-local terms to arise. The solution for the streamfunction $\psi$ defined by $\psi$ by $U_{1}=-(1 r) \psi_{z}, W_{1}=(1 r) \psi_{r}$ is given in Fourier space by (see Papageorgiou et al. 1990 for details)

$$
\widehat{\psi}=C_{1}(k) r I_{1}(k r)+C_{2}(k) r^{2} I_{0}(k r),
$$

where $I_{0}, I_{1}$ are the modified Bessel functions of order zero and one, respectively, and $C_{1}(k), C_{2}(k)$ are functions of $k$ to be found. (The Fourier transform is defined in the usual way for a function $\chi(r, z, t)$ to be $\left.\widehat{\chi}=\int_{-\infty}^{\infty} \chi(r, z) e^{-i k z} d z.\right)$

The Fourier transform of $A$ is found by expressing the tangential stress equation (3.26) in terms of $\psi$, taking the Fourier transform in $z$ and using the solution (3.32) at $r=1$, to obtain

$$
\widehat{A}=-\frac{2 k}{\lambda}\left(k C_{1}+C_{2}\right) I_{1}(k)-\frac{2 C_{2} k^{2}}{\lambda} I_{0}(k)-\frac{i k \beta_{0}}{\lambda C_{a 0}} \widehat{\tilde{\Gamma}}-i k \widehat{P_{20}}
$$

Taking the Fourier transform of $U_{2}(y, z, t)$ in $(3.24)$ and using (3.33), yields

$$
\widehat{U}_{2}(y, k, t)=\frac{\lambda k^{2}}{2 m}\left(1-\frac{1}{3} y\right) y^{2} \widehat{P}_{20}-\frac{i k^{2}\left(k C_{1}+C_{2}\right)}{m} y^{2} I_{1}(k)-\frac{i k^{3} C_{2}}{m} y^{2} I_{0}(k)+\frac{k^{2} \beta_{0}}{2 m C_{a 0}} y^{2} \widehat{\tilde{\Gamma}} .
$$

Finally, $C_{1}(k)$ and $C_{2}(k)$ are determined in terms of $\hat{H}$ by inserting (3.32) into the Fourier transform of the continuity of velocity boundary conditions (3.28) (see Papageorgiou et al. 1990). After evaluating (3.34) at $y=1$ and applying the inverse Fourier transform, we obtain

$$
U_{2}(1, z, t)=-\frac{i}{m \pi}\left(1-\frac{1}{m}\right) \int_{-\infty}^{\infty} N(k) \hat{H}(k) e^{i k z} d k-\frac{\lambda}{3 m} P_{20 z z}-\frac{\beta_{0}}{2 m C_{a 0}} \tilde{\Gamma}_{z z}
$$

where $N(k)=\frac{k^{2} I_{1}^{2}(k)}{k I_{1}^{2}(k)-k I_{0}^{2}(k)+2 I_{0}(k) I_{1}(k)}$. The desired evolution equation is obtained by using (3.35) in the kinematic condition (3.27) and the elimination of $P_{20}$ in favor of $H$ through (3.25), and is given by

$$
\begin{aligned}
H_{\tau} & -\frac{2}{m} H H_{z}+\frac{i}{m \pi}\left(1-\frac{1}{m}\right) \int_{-\infty}^{\infty} N(k) \int_{-\infty}^{\infty} H(z, \tau) e^{i k(z-\tilde{z})} d \tilde{z} d k \\
& +\frac{J}{3 m \lambda}\left(H+H_{z z}\right)_{z z}+\frac{\beta_{0}}{2 m C_{a 0}} \tilde{\Gamma}_{z z}=0
\end{aligned}
$$

Equation (3.36) without the surfactant term is identical to equation (28) in Papageorgiou et al. (1990). The new term comes from the tangential stress balance (2.18) and is the Marangoni force (the parameter $\beta_{0} / C_{a 0}$ is the Marangoni number defined in the usual way). The integral term represents the influence of viscosity stratification; when $m=1$ that term disappears. Note that for $m=1$ and $\beta_{0}=0$, the equation reduces to the Kuramoto-Sivashinsky equation (see Frenkel et al. (1987), Papageorgiou et al. (1990)).

Finally for simplicity, the surfactant equation (3.16) is written as

$$
\Gamma_{\tau}-\frac{2}{m}(\Gamma H)_{z}=\frac{1}{\bar{P}_{e}} \Gamma_{z z}
$$

where $\Gamma=\bar{\Gamma}_{0}+\tilde{\Gamma}$. The coupled system (3.36) (with $\tilde{\Gamma}$ replaced by $\Gamma$ ) and (3.37) is studied numerically in section 5 . In what follows the overline is dropped from $\bar{\Gamma}_{0}$. 


\section{Canonical rescaling and linear stability}

The evolution equations (3.36) and (3.37) are rescaled using the transformations

$$
z \rightarrow-z, \quad H \rightarrow \frac{J}{6 \lambda} H, \quad \tau \rightarrow \frac{3 m \lambda}{J} t, \quad \Gamma \rightarrow \frac{J^{2} C_{a 0}}{9 \lambda^{2} \beta_{0}} \Gamma,
$$

to give the following canonical system

$$
\begin{gathered}
H_{t}+H H_{z}+i \Lambda\left(\int_{-\infty}^{\infty} N(k) \int_{-\infty}^{\infty} H(\tilde{z}, \tau) e^{i k(z-\tilde{z})} d \tilde{z} d k\right)+H_{z z}+H_{z z z z}+\Gamma_{z z}=0 \\
\Gamma_{t}+(H \Gamma)_{z}-\eta \Gamma_{z z}=0
\end{gathered}
$$

where

$$
\Lambda=-\frac{1}{\pi}\left(1-\frac{1}{m}\right) \frac{3 \lambda}{J}, \quad \text { and } \quad \eta=\frac{3 m \lambda}{J \bar{P}_{e}} \geq 0
$$

The parameter $\Lambda$ is negative when $m>1$ (film more viscous than core) and positive when $m<1$ (film less viscous than core). The linearized form of equations (4.2)-(4.3) when $\Lambda=0$ recovers the system of Wei \& Rumschitzki as detailed below.

Linearizing (4.2)-(4.3) about the uniform states $H=0, \Gamma=\Gamma_{0}$, and looking for normal mode solutions proportional to $\exp (i k z+\omega t)$, yields the following dispersion relation

$$
\omega^{2}+\left[k^{4}-k^{2}+\eta k^{2}+i \Lambda N(k)\right] \omega+i \eta \Lambda k^{2} N(k)+\eta k^{2}\left(k^{4}-1\right)+i \Gamma_{0} k^{3}=0 .
$$

The solution of (4.5) yields two roots given by

$$
\begin{aligned}
2 \omega(k) & =-\left(\eta k^{2}+k^{4}-k^{2}+i \Lambda N(k)\right) \\
& \pm \sqrt{\left(\eta k^{2}+k^{4}-k^{2}+i \Lambda N(k)\right)^{2}-4 i \eta \Lambda k^{2} N(k)-4 \eta k^{2}\left(k^{4}-k^{2}\right)-4 i \Gamma_{0} k^{3}},
\end{aligned}
$$

and the flow is linearly unstable if $\mathcal{R}(\omega(k))=\omega_{R}(k)>0$ for any $k$. An apparent difference with the clean case is that for $\Gamma_{0} \neq 0$, we generally have $\omega_{I} \neq 0$, implying dispersion as well as growth/damping. It can be anticipated, therefore, that for parameter values where the KS equation has non-uniform steady states, the presence of surfactant will induce a travelling wave. This feature, among others, is explored fully using numerical experiments in section 5 .

The dispersion relation (4.6) can be obtained from that of the full linear problem by taking an appropriate thin annular limit. This has been shown for the surfactant-free case by Georgiou et al. (1992) and details for the case with surfactant will be provided elsewhere. The full linear problem has been calculated by Blyth et al. (2006) and we note that the present analysis recovers the neutral stability curve in the vicinity of the origin of their Figure 8 in the thin annular limit. Furthermore the analysis here extends the dynamics into the nonlinear regime for flows having thin annuli which are difficult to resolve using direct numerical simulations.

Before providing typical growth rate characteristics we consider some limiting forms. We recover the surfactant-free limit analyzed by Papageorgiou et al. (1990) and Georgiou et al. (1992) by considering $\eta \gg 1$ in the dispersion relation (4.6) (equivalently, it can be done asymptotically in the perturbation PDEs by utilizing the ordering $H=O(1)$ and $\Gamma=O(1 / \eta))$. A large $\eta$ ordering is physically relevant since it corresponds to very large interfacial diffusion of the insoluble surfactant, and hence in a uniform distribution of surfactant and surface tension coefficient. The result is

$$
\omega_{+}=k^{2}-k^{4}-i \Lambda N(k)+O(1 / \eta), \quad \omega_{-}=-\eta k^{2}+O(1)
$$

The leading order part of the root $\omega_{+}$corresponds to the Kuramoto-Sivashinsky case when viscosity stratification is present (i.e. equation (4.2) in the absence of $\Gamma$ ). Analogous results hold when $|\Lambda|$ is large (note that $-\frac{3 \lambda}{\pi J}<\Lambda<\infty$ but we can consider a large negative lower bound to obtain the asymptotic behavior). As before, there is a growing and a damped root with asymptotic forms, as $|\Lambda| \rightarrow \infty$,

$$
\omega_{+}=k^{2}-k^{4}+-i \Lambda N(k)+O(1 /|\Lambda|), \quad \omega_{-}=-\eta k^{2}+O(1 /|\Lambda|) .
$$




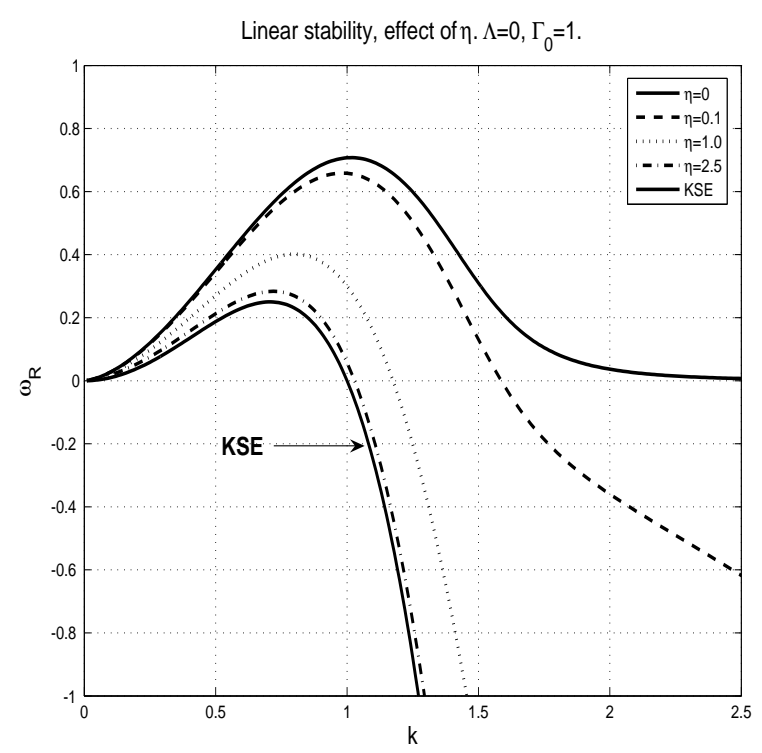

Figure 1. Effect of $\eta$ on linear growth rates; $\Lambda=0, \Gamma_{0}=1$. Values of $\eta$ are shown in the figure.

The leading order growth rate is identical to that of the KS equation in the absence of surfactant. A physical explanation for this is the following: when $|\Lambda|$ is large the shear jump between film and core is large and overcomes any shear stresses set up by Marangoni forces. Thus the interface behaves to leading order as if it were clean.

At large $\eta$ and $\Lambda$, then, the stability tends to that for a clean interface. Next, we give results for intermediate values and choose to fix $\Gamma_{0}=1$. In figure 1 we show the effect of increasing the surfactant diffusion coefficient $\eta$ when $\Lambda=0$. The growing mode alone is indicated - the other root is always damped. It is seen that when $\eta=0$ the flow is unstable for all wavenumbers but the growth rate decreases to zero as $k \rightarrow \infty$. At non-zero $\eta$, the infinite band of unstable waves becomes finite and the growth rate curve decreases monotonically to the KS equation curve as shown by (4.7).

The effect of $\eta$ on the stability characteristics when $\Lambda=1$ and $\Lambda=-1$ are shown in figures 2 and 3 . In the former case (i.e. core less viscous than the annulus), the effect of the surfactant is to enhance the instability for all values of $\eta$ relative to the clean case. The growth rate corresponding to the root $\omega_{+}$is higher than that for KS equation for all values of $k$, and tends asymptotically to the KS equation curve as $\eta \rightarrow \infty$, as established already in (4.7). The second root, $\omega_{-}$always provides damping, but we note that when $\eta=0$ the corresponding growth rate tends to zero from below as $k \rightarrow \infty$. The monotonic convergence of the growth rate $\omega_{+}$to the corresponding KS equation curve is shown collectively in the bottom right panel of figure 2 . When $\Lambda=-1$ (i.e. the core is more viscous than the annulus), the presence of the surfactant enhances the instability for sufficiently small values of $\eta$. In contrast to the $\Lambda=1$ case, both roots are growing modes when $\eta=0$ (top left panel of figure 3), with $\omega_{+}$and $\omega_{-}$being more and less unstable than the KS equation case, respectively. As $\eta$ increases from zero, both the maximum growth rates and the length of the band of unstable wavenumbers for $\omega_{+}$and $\omega_{-}$decrease. In particular when $\eta=0.5$ (top right panel), the $\omega_{-}$mode is completely stable and the $\omega_{+}$has a significantly reduced growth rate and band of unstable wavenumbers. As $\eta$ increases further, the $\omega_{-}$mode becomes increasingly stable, whereas the $\omega_{+}$mode exhibits a nonmonotonic behavior as it becomes more unstable and asymptotes to the KS equation curve as $\eta \rightarrow \infty$. The effect of varying $\eta$ has is summarized in figure 4 which plots the neutral curves of $k$ versus $\eta$ for fixed negative $\Lambda=-1$ and initial surfactant concentration $\Gamma_{0}=1$. The wavenumber $k=0$ is always neutral and the solid line depicts the variation of the non-zero neutral wavenumber with $\eta$. Regions above this curve are stable (short waves) and the region below is unstable as indicated. As $\eta$ increases from zero the band of unstable wavenumbers decreases (when $\eta=0$ there is instability for all $k$ - see for example figure 3) and follows the $\omega_{+}$mode initially. At a value of $\eta \approx 0.5$ there is mode-crossing and the $\omega_{+}$mode becomes stable while the $\omega_{-}$mode takes and the neutral stability curve asymptotes to $k=1$ as already pointed out in the large $\eta$ analysis in equation (4.7). 

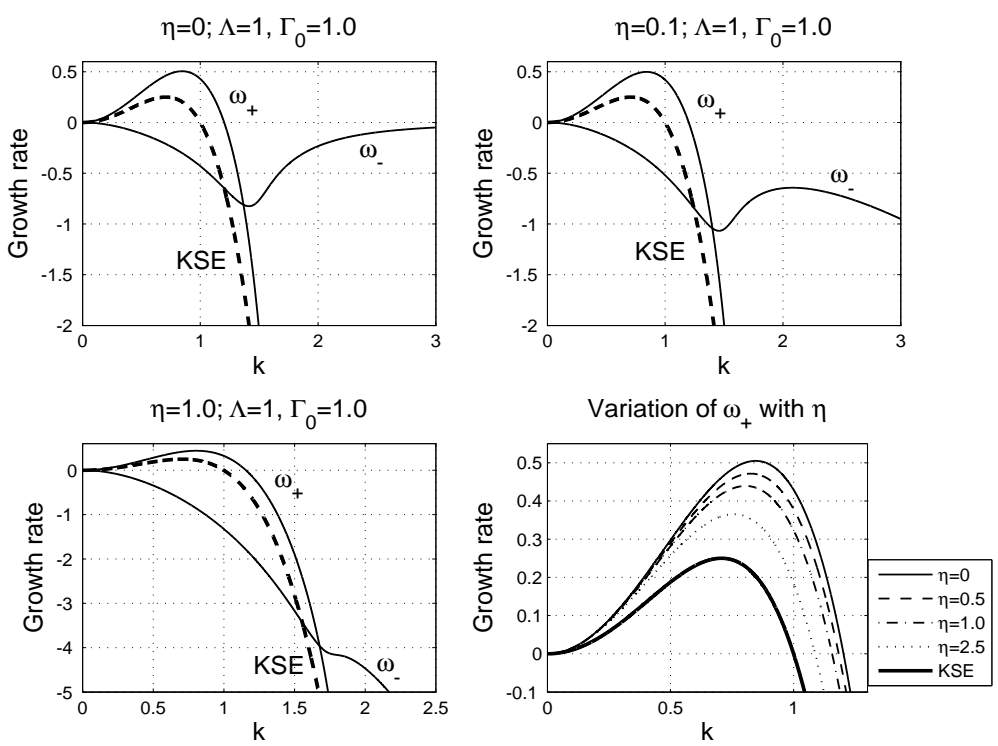

Figure 2. Effect of $\eta$ on linear growth rates; $\Lambda=1, \Gamma_{0}=1$. Values of $\eta$ are shown in the figure.

We consider next the instability characteristics as $\Lambda$ is varied for fixed $\eta=0.5$ and $\Gamma_{0}=1$. It is found that for $\Lambda>0$ one mode is unstable $\left(\omega_{+}\right)$, while the other mode $\omega_{-}$is always damped. The instability induced by the presence of surfactant when $\Lambda=0$ is reduced by increasing $\Lambda$ (i.e. increasing the core viscosity relative to that of the film) and eventually the the growth rate curve asymptotes to that for a clean interface as $\Lambda \rightarrow \infty$ as established in (4.8). The situation for negative $\Lambda$ is different (figure 5). Both modes can be unstable and in fact an exchange takes place as $\Lambda$ decreases; When $\Lambda=0$, the $\omega_{+}$mode lies above the KS equation curve whereas the $\omega_{-}$mode is stable for all wavenumbers and lies below the KS mode (top left panel). As $\Lambda$ decreases, the $\omega_{+}$mode becomes increasingly stable and the $\omega_{-}$mode increasingly unstable (see top right panel, $\Lambda=-0.5$ ). By $\Lambda=-1$ the $\omega_{+}$mode is completely stable while the $\omega_{-}$mode becomes unstable for a range of wavenumbers starting at $k=0$ - see bottom left panel. As $\Lambda$ decreases further, the $\omega_{+}$mode becomes increasingly more stable while the $\omega_{-}$mode asymptotes to the clean KS equation curve as established by the result (4.8) (see bottom right panel, $\Lambda=-10$ ). In summary, then, a large viscosity contrast ( $\Lambda$ positive or negative) decreases the (normally destabilizing) effect of surfactants. In figure 6 we provide the neutral stability characteristics for fixed $\eta=0.5$ and $\Gamma_{0}=1$. Once again there is mode crossing so that the unstable $\omega_{-}$mode which dominates for $\Lambda<0$ is overtaken by the $\omega_{+}$mode which is unstable for $\Lambda>0$. The dashed lines in the figure follow the continuation of the modes after crossing. It can be seen, for example, that in the region bounded by the dashed curves, both modes are unstable but the solid curve gives the largest unstable value of $k$. We also note that both branches of the neutral stability curve asymptote to $k=1$ as $|\Lambda| \rightarrow \infty$ as anticipated by the asymptotic result (4.8).

We conclude this section by considering a useful localization of the linear pseudo-differential operator that appears in (4.2). Following Smyrlis \& Papageorgiou (1998), we note that $N(k)$ is an odd function of $k$ and hence the operator has a purely imaginary symbol in Fourier space, corresponding to a generalized odd derivative. We approximate $N(k)$ by its two-terms Taylor expansion $N(k)=2 k+\frac{1}{6} k^{3}$, which casts equation (4.2) into a $\mathrm{KS}-\mathrm{KdV}$ type equation

$$
H_{t}+H H_{z}+2 \Lambda H_{z}-\frac{\Lambda}{6} H_{z z z}+H_{z z}+H_{z z z z}+\Gamma_{z z}=0
$$

to be solved along with (4.3). In this case the dispersion relation (4.6) holds with $N(k)$ given by its two-terms expansion. We have re-calculated all instability results presented in figures 2-5 using the above local approximation, and found them to be almost identical. Numerical calculations of Smyrlis \& Papageorgiou Smyrlis \& Papageorgiou(1998) of the nonlinear problem show that the localized equations are a good approximation to the full non-local problem. In the numerical work that follows we concentrate on the case $\Lambda=0$, but also give representative results for non-zero $\Lambda$ using the localized system (4.9). 

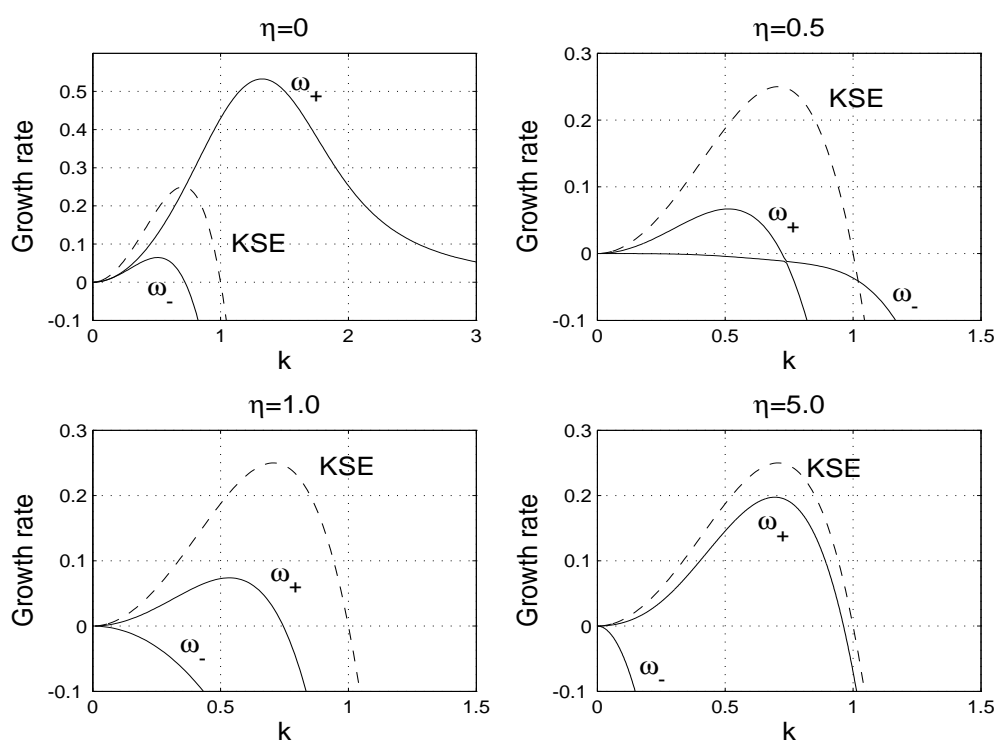

Figure 3. Effect of $\eta$ on linear growth rates; $\Lambda=-1, \Gamma_{0}=1$. Values of $\eta$ are shown in the figure.

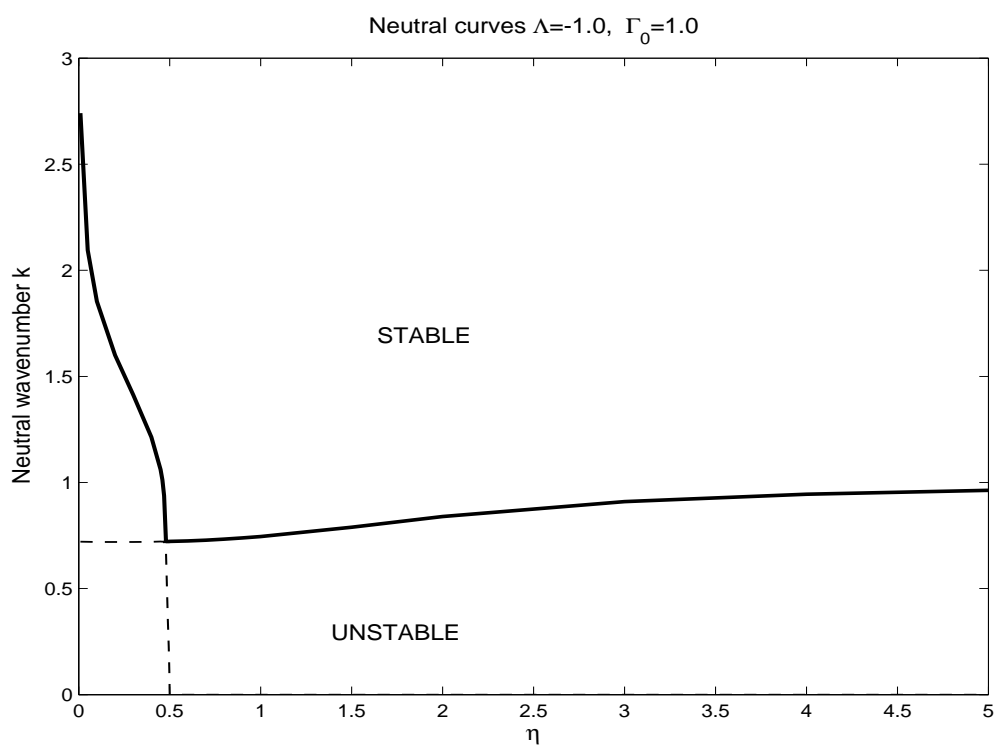

FiguRE 4. Effect of $\eta$ on linear growth rates; $\Lambda=-1, \Gamma_{0}=1$. Values of $\eta$ are shown in the figure.

\section{Numerical Methods}

In this section we solve the system (4.2)-(4.3) numerically on periodic domains of period $2 L$. The value of $L$ is a parameter measuring the size of the system and as $L$ increases more unstable modes enter into the dynamics. On $2 L$-periodic domains, the non-local term in (4.2) has the form of a Fourier series, namely

$$
i \Lambda \sum_{n=-\infty}^{\infty} N(\pi k / L)\left(\frac{1}{2 L} \int_{-L}^{L} H(\tilde{z}, t) \mathrm{e}^{-i n \pi \tilde{z} / L}\right) \mathrm{e}^{i n \pi \tilde{z} / L}
$$

Following Papageorgiou \& Smyrlis (1991), Smyrlis \& Papageorgiou (1991), (1996), (1998), we re-scale the problem to a $2 \pi$-periodic domain. The appropriate transformations are (for brevity the same symbols are 

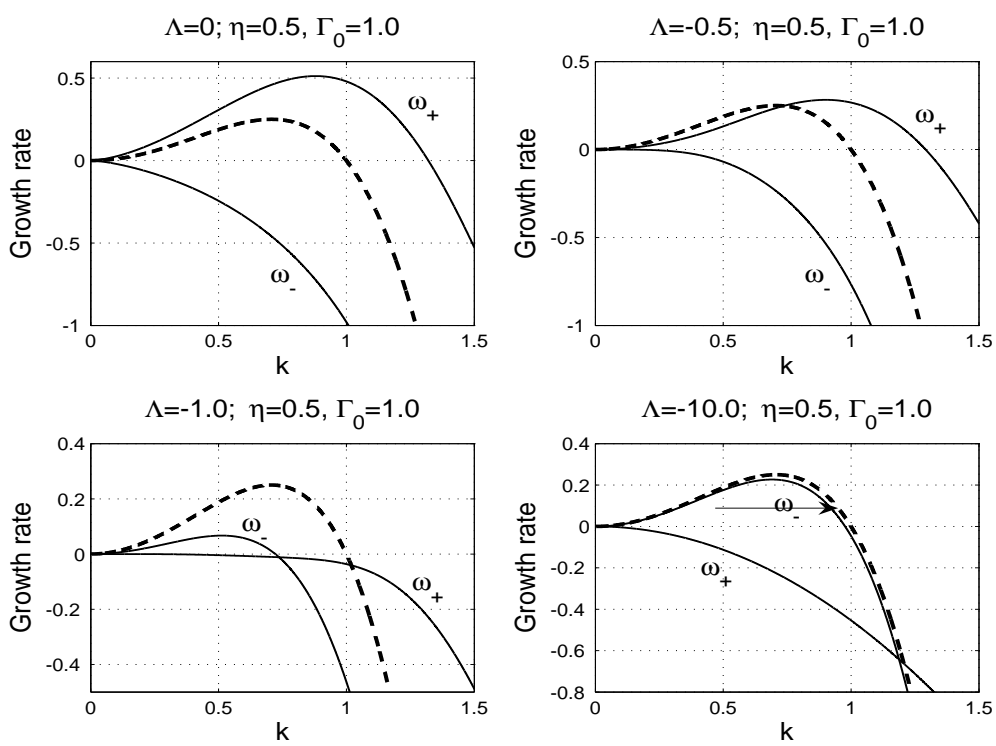

Figure 5. Effect of $\Lambda<0$ on linear growth rates; $\eta=0.5, \Gamma_{0}=1$. Values of $\Lambda$ are shown in the figure. The dashed curve corresponds to the clean case.

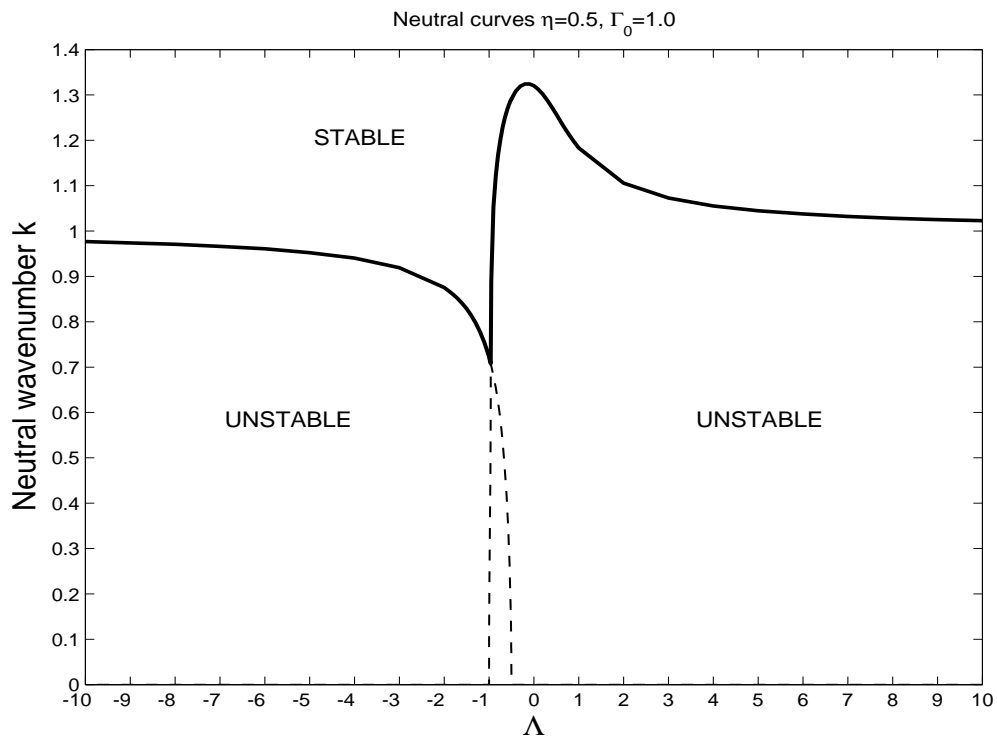

FiguRE 6. Effect of $\Lambda<0$ on linear growth rates; $\eta=0.5, \Gamma_{0}=1$. Values of $\Lambda$ are shown in the figure. The dashed curve corresponds to the clean case.

used):

$$
z \rightarrow \frac{L}{\pi} z, \quad t \rightarrow \frac{L}{\pi} t, \quad H \rightarrow \frac{\pi}{L} H, \quad \Gamma \rightarrow \frac{\pi}{L} \Gamma .
$$

Defining the positive parameter

$$
\nu=\frac{\pi^{2}}{L^{2}}
$$

leads to the following canonical system

$$
H_{t}+H H_{z}+i \Lambda \sum_{k=-\infty}^{\infty} N(k \sqrt{\nu}) \widehat{H}_{n} \mathrm{e}^{i k z}+H_{z z}+\nu H_{z z z z}+\Gamma_{z z}=0
$$




$$
\Gamma_{t}+(H \Gamma)_{z}-\eta \Gamma_{z z}=0
$$

where $\widehat{H}_{n}=(1 / 2 \pi) \int_{-\pi}^{\pi} H(\xi, t) \mathrm{e}^{-i n \xi} d \xi$ are the Fourier coefficients of $H(z, t)$ on the given interval. We note that the analogous re-scaling applied to the localized system transforms equation (4.9) into

$$
H_{t}+H H_{z}+2 \frac{\Lambda}{\sqrt{\nu}} H_{z}-\frac{\Lambda \sqrt{\nu}}{6} H_{z z z}+H_{z z}+\nu H_{z z z z}+\Gamma_{z z}=0
$$

When $\Gamma$ is absent, the transformations $x \rightarrow-x, H \rightarrow-H$ and $\Lambda \rightarrow-\Lambda$ make it possible to obtain solutions corresponding to $\Lambda<0$ from those for $\Lambda>0$. This is not the case when $\Gamma$ is present due to the coupling term $\Gamma_{z z}$.

It follows directly from the system (5.4) (or (5.6)) and (5.5) that $\int_{0}^{2 \pi} H d z$ and $\int_{0}^{2 \pi} \Gamma d z$ are conserved quantities and can be used as an accuracy check on the numerics. In particular, defining initial conditions

$$
H(z, 0)=H_{0}(z), \quad \Gamma(z, 0)=G_{0}(z),
$$

with $(1 / 2 \pi) \int_{0}^{2 \pi} H_{0}(z) d z=0$ and $(1 / 2 \pi) \int_{0}^{2 \pi} G_{0}(z) d z=\Gamma_{0}>0$, we have the conserved quantities

$$
\int_{0}^{2 \pi} H(z, t) d z=0 \quad \text { and } \quad \int_{0}^{2 \pi} \Gamma(z, t) d z=2 \pi \Gamma_{0} .
$$

The numerical scheme employed here is implicit and pseudo-spectral. Taking the Fourier transform of the system (the symbol $\mathcal{F}$ will be used to denote the Fourier transform operation) (5.4)-(5.5) and defining the complex valued vector $\mathbf{u}_{k}(t)=\left(\widehat{H}_{k}(t), \widehat{\Gamma}_{k}(t)\right)^{T}$, where hats denote Fourier transforms, $k$ is the wavenumber and a superscript $T$ denotes the transpose of a vector, casts the system into the following continuous time equation

$$
\frac{d \mathbf{u}_{k}}{d t}+M(k) \mathbf{u}_{k}=\mathbf{f}_{k}
$$

where $\mathbf{f}_{k}=\left(f_{k}^{(1)}, f_{k}^{(2)}\right)^{T}$ with $f_{k}^{(1)}(t)=-\mathcal{F}\left[H H_{z}\right](t ; k), f_{k}^{(2)}(t)=-\mathcal{F}\left[(H \Gamma)_{z}\right](t ; k)$ and the $2 \times 2$ matrix $M(k)$ given by

$$
M(k)=\left(\begin{array}{cc}
\nu k^{4}-k^{2}+i \Lambda N(k \sqrt{\nu}) & -k^{2} \\
0 & \eta k^{2}
\end{array}\right) .
$$

Note that in the case of the local equation (5.6) the symbol $N(k \sqrt{\nu})$ in the matrix $(5.9)$ becomes $N(p)=$ $\frac{2 k \sqrt{\nu}}{\nu}+\frac{(k \sqrt{\nu})^{3}}{6 \nu}$. Discretizing in time so that $\mathbf{u}_{k}^{n}$ is the numerical approximation of $\mathbf{u}_{k}(n \Delta t)$ where $\Delta t$ is the time-step, we propose the following two-step scheme

$$
\begin{aligned}
& \frac{\mathbf{u}_{k}^{n+1 / 2}-\mathbf{u}_{k}^{n}}{(\Delta t / 2)}+M(k) \mathbf{u}_{k}^{n+1 / 2}=\mathbf{f}_{k}^{n}, \\
& \frac{\mathbf{u}_{k}^{n+1}-\mathbf{u}_{k}^{n}}{\Delta t}+\frac{1}{2} M(k)\left(\mathbf{u}_{k}^{n+1}+\mathbf{u}_{k}^{n}\right)=\mathbf{f}_{k}^{n+1 / 2} .
\end{aligned}
$$

It can be shown (omitted for brevity) that the scheme is of accuracy $\mathcal{O}\left(\Delta t^{2}\right)$ in time (and spectral in space). For each value of $k$ the system can be solved analytically to obtain $\mathbf{u}_{k}^{n+1 / 2}$ from (5.10) The vector $\mathbf{u}_{k}^{n+1 / 2}$ is inserted into (5.11) which is solved explicitly for $\mathbf{u}_{k}^{n+1}$, resulting in a two-step scheme (the formulas are not given for brevity). All transforms are performed using the Fast Fourier Transform (FFT) and inverse FFTs are used to obtain function values in real space as needed. More details can be found in Kas-Danouche (2002).

Due to the complex dynamical phenomena we utilize several tools in the evaluation of the different attractors found numerically. One useful tool is the $L^{2}$-norm (or energy) of the solution $H(z, t)$ defined by

$$
E(t)=\|H(\cdot, t)\|_{2}^{2}=\int_{0}^{2 \pi} H(z, t)^{2} d z
$$

This is used in the construction of the phase-plane $(E(t), d E(t) / d t)$ and the particular Poincaré sections satisfying $d E / d t=0$. The latter points are the maxima and minima of the graph of $E(t)$. We denote the maxima of $E(t)$ by the pairs $\left(t_{j}, E_{j}\right)_{j=1, \infty}$ (analogous notations hold for the minima). The points $\left(E_{j}, E_{j+1}\right), j=1, \ldots$, in $\mathbf{R}^{2}$ represent the flow generated by the infinite-dimensional dynamical system using the $L^{2}$ projection. 

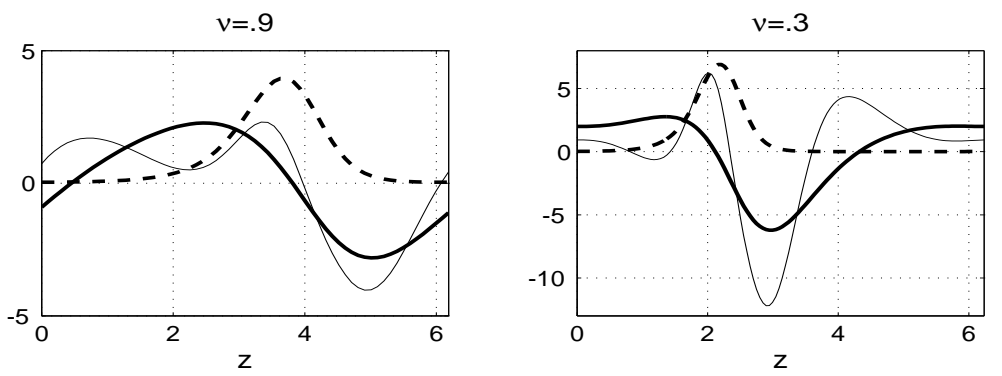

$v=.095$
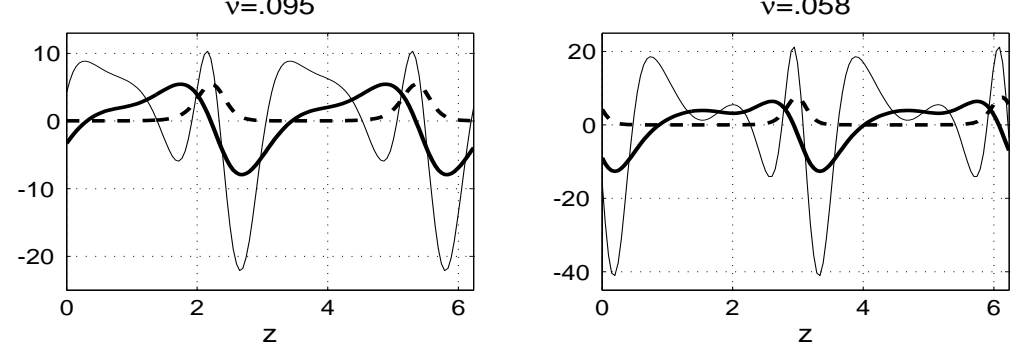

FiguRE 7. Effect of $\nu$ (values shown) on profiles of interfacial shape $H$ (thick solid line), surfactant concentration $\Gamma$ (dashed), and axial velocity $W_{2}$ (light solid line); $\Lambda=0, \Gamma_{0}=1.0$. Top panels fully modal (window $A_{1}$ ), bottom panels bi-modal (window $A_{2}$ ).

The union of these points is a set $\mathcal{A}$ which can provide insights into the dynamics. For example, if there is only one point in $\mathcal{A}$ then the flow is seen to be time-periodic with one minimum (and one maximum); two points in $\mathcal{A}$ imply time-periodicity with two maxima (and two minima), and so on. Quasi-periodicity and chaotic dynamics are also suggested by this construction which is a return map; in the former case by curves in $\mathbf{R}^{2}$ which become dense (i.e. non-fractal) as the evolution develops, and in the latter by fractal curves, folding and self-similarity. From a computational viewpoint we emphasize that the construction of such return maps is typically expensive since the PDE must be integrated over long time intervals to add a point (as in period-doubling cascades, for example), or if the flow is chaotic, over long periods of time to visit the same vicinity of the plane. More details on the use of numerical return maps in estimating the universal Feigenbaum constants for the Kuramoto-Sivashinsky equation can be found in Papageorgiou \& Smyrlis (1991) and Smyrlis \& Papageorgiou (1991), (1996).

\section{Numerical Results}

There are four parameters present in the evolution equations, the "viscosity" parameter $\nu>0$, the viscosity stratification parameter $\Lambda$, the surfactant diffusion coefficient $\eta>0$ and the initial surfactant concentration $\Gamma_{0}>0$. The possible long time behavior of solutions includes stationary and travelling wave attractors, as well as time-periodic/quasi-periodic and chaotic attractors. In order to determine the nature of the solution over long times, we have performed extensive numerical experiments, examining hundreds of values of the parameters $\nu, \Lambda, \eta$ and $\Gamma_{0}$. We give representative solutions rather than completely mapping out the phase space. Our numerical results are based on solutions of the initial value problem and we therefore calculate stable or attracting states rather than a complete picture of stable and unstable solution branches.

\subsection{Equal viscosities $\Lambda=0$}

This case corresponds to the insoluble surfactant generalization of the KS equation. The initial conditions for the results that follow are

$$
H(z, 0)=-\sin (z), \quad \Gamma(z, 0)=\Gamma_{0}=1,
$$

and to begin with we fix $\eta=1$ and consider decreasing values of $\nu$ (i.e. we increase the system size). For $\nu$ larger than approximately 1.47 the long time evolution gives a uniform trivial state and nontrivial steady-state travelling waves emerge below this value. The nontrivial waves are initially fully modal (i.e. 


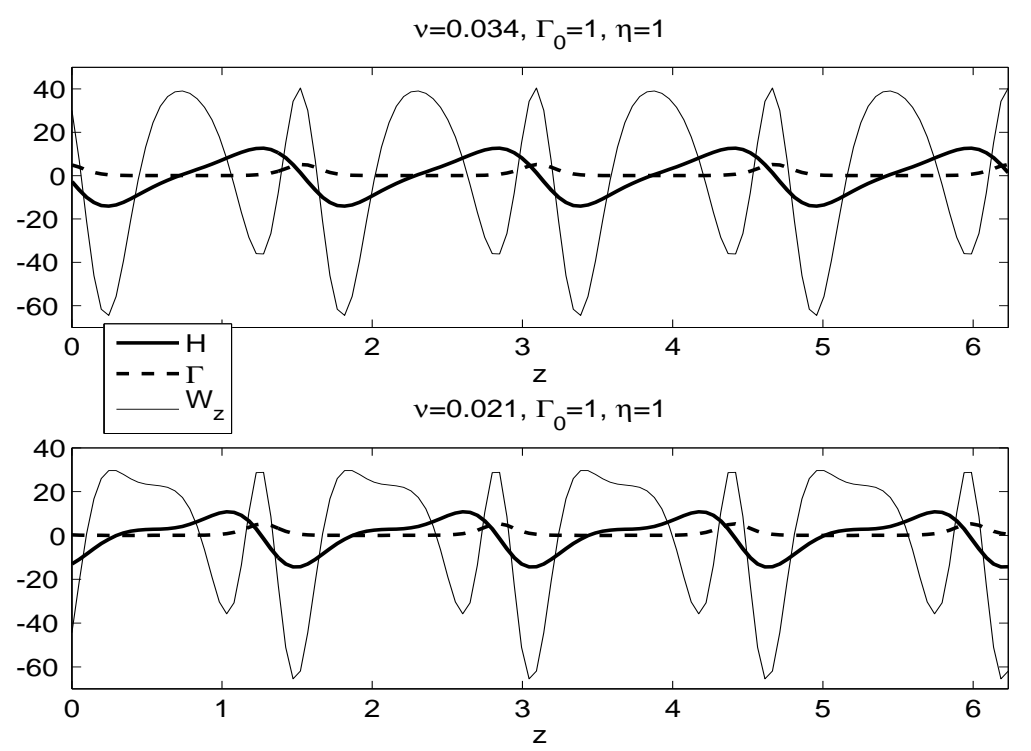

FIGURE 8. Effect of $\nu$ (values shown) on tetra-modal travelling wave profiles (window $A_{4}$ ) of interfacial shape $H$ (thick solid line), surfactant concentration $\Gamma$ (dashed), and axial velocity $W_{2}$ (light solid line); $\Lambda=0, \Gamma_{0}=1.0$.
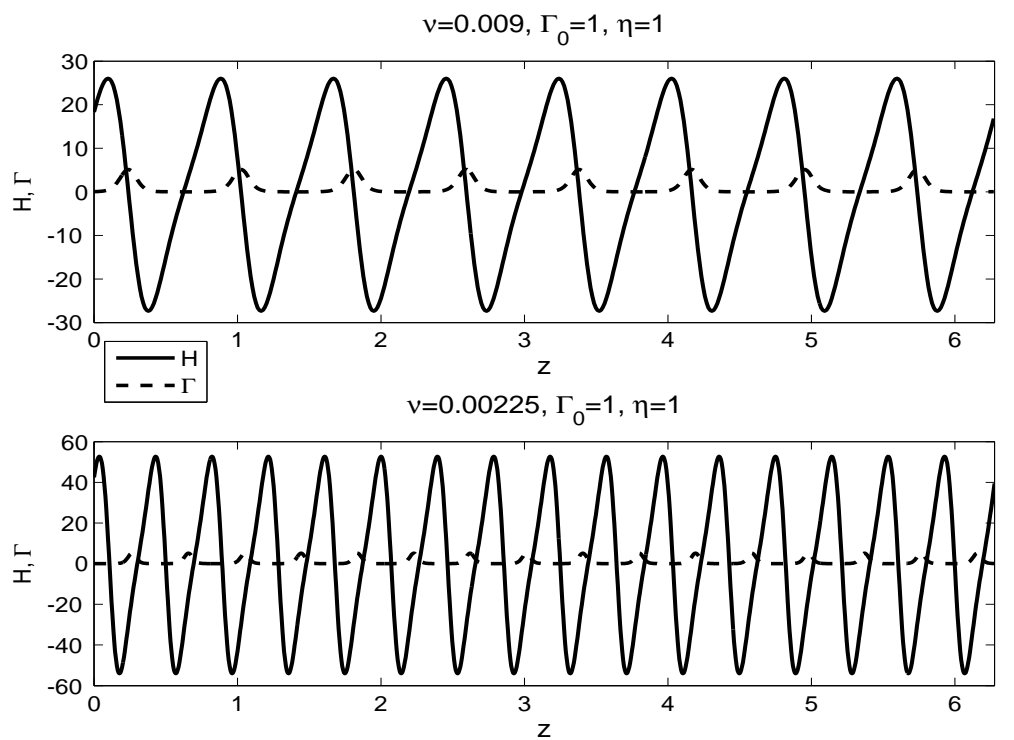

Figure 9. Effect of $\nu$ (values shown) on profiles of interfacial shape $H$ (thick solid line), and surfactant concentration $\Gamma$ (dashed); $\Lambda=0, \Gamma_{0}=1.0$. Top panel octa-modal window $A_{8}$, bottom panels decahexa-modal window $A_{16}$.

they contain all Fourier modes) for $\nu \geq 0.2$, and have decreasing speeds and increasing $L^{2}$ norms. The travelling wave speed changes sign at a value of $\nu$ between 0.5 and 0.4 , and remains negative and decreasing as noted above. At the value $\nu=0.19$ (approximately), a Hopf bifurcation occurs giving rise to time-periodic travelling waves, i.e., waves which after a full period of oscillation take on the shape at the start of the period but shifted spatially. Using the energy (5.12) and the Poincare sections described above, we find that on entering this first time-periodic attractor, $E(t)$ has one maximum and one minimum as $\nu$ decreases from 0.19 to 0.116 . The period of oscillation decreases from approximately 1.380 to 1.097 , respectively. At a value of $\nu$ between 0.116 and 0.115 , a bifurcation occurs that produces three maxima and three minima in the graph of $E(t)$; this situation persists until a value of $\nu$ between 0.112 and 0.111 , at which a further bifurcation gives rise to oscillations with five maxima and minima. This sub-window persists until $\nu \approx 0.099$, 


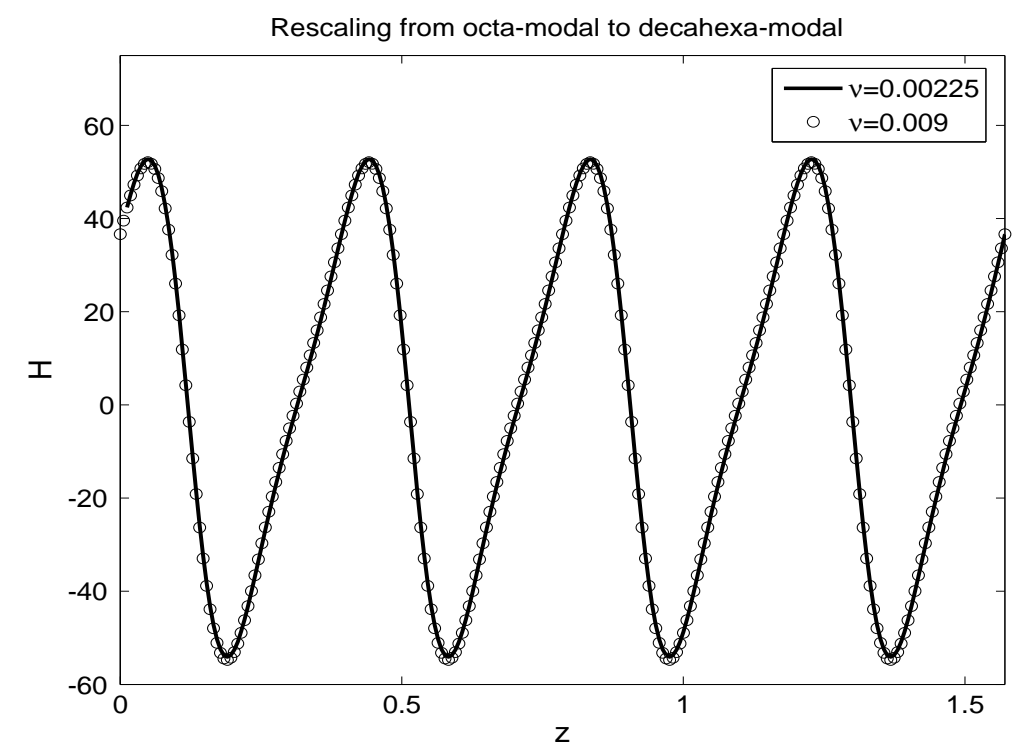

FIgURE 10. Re-scaling of the interfacial shape $(H)$ octa-modal solutions (top panel of figure 9 ) to the decahexa-modal solutions (bottom panel of figure 9) using the asymptotic similarity property (6.2). Solid line, deca-hexamodal solution at $\nu=0.00225$; circles, re-scaled octa-modal solution at $\nu=0.009$. Only part of the wave is shown.

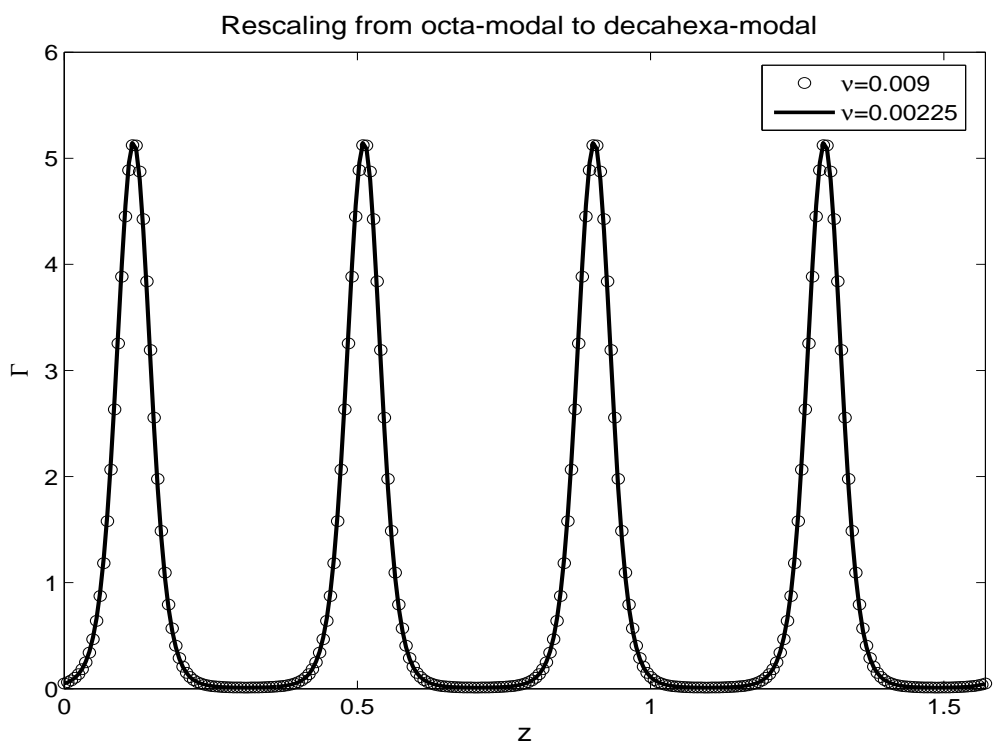

FiguRE 11. Re-scaling of the surfactant distribution $(\Gamma)$ octa-modal solutions (top panel of figure 9) to the decahexamodal solutions (bottom panel of figure 9) using the asymptotic similarity property (6.2). Solid line, deca-hexamodal solution at $\nu=0.00225$; circles, re-scaled octa-modal solution at $\nu=0.009$. Only part of the wave is shown.

below which bi-modal steady-state travelling waves emerge; these are waves with non-zero Fourier modes $k=2 n$ for integer $n$. The bifurcations in the time-periodic window are not smooth as would be the case in a period-doubling, for instance. This has been confirmed numerically by monitoring the average of $E$ over a period, defined as $I_{E}(\nu)=(1 / T) \int_{t}^{t+T} E(s ; \nu) d s$, where $t$ is any chosen time (sufficiently large for transients to be irrelevant) and $T$ is the period of oscillation. For example, in crossing from $\nu=0.116$ to $\nu=0.115$ (from the first periodic sub-window to the second), $I_{E}$ jumps from 11.761 to 18.085 , while crossing from the second sub-window to the third $I_{E}$ jumps from a value of 20.273 at $\nu=0.112$ to 56.396 at $\nu=0.111$ (see Blyth, Hall \& Papageorgiou (2003) for similar phenomena in pulsating cylindrical flows).

With a further decrease of $\nu$, the bimodal steady-state travelling waves loose stability through a Hopf 


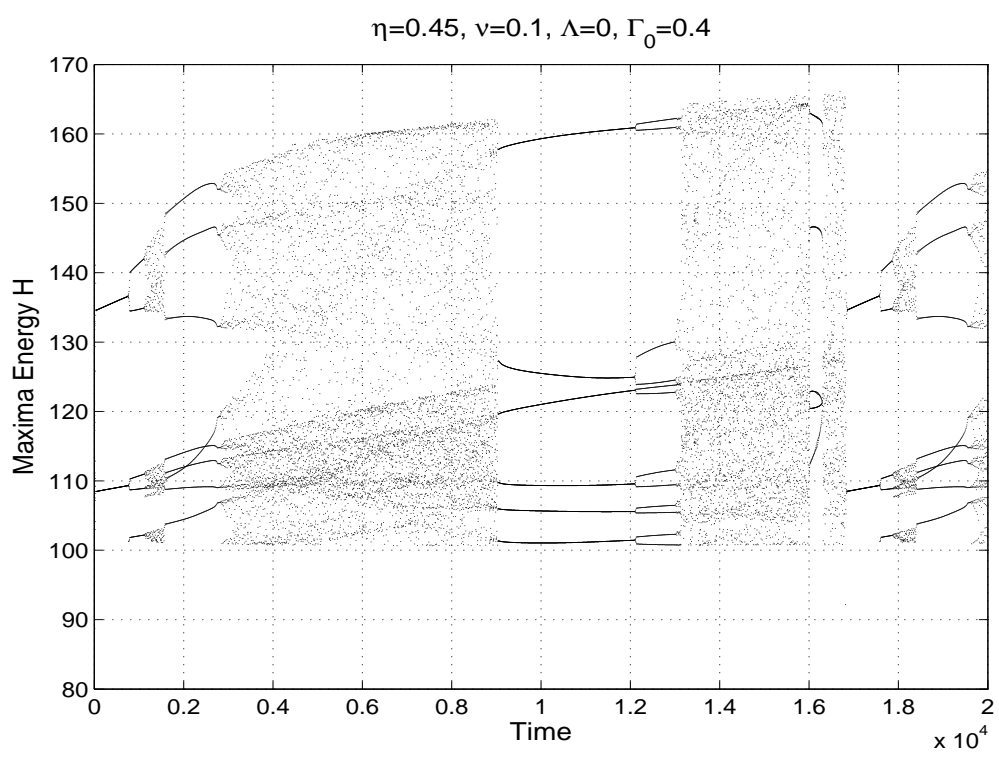

Figure 12. The evolution of the maxima of $E(t)=\|H\|_{2}^{2}$ for $\eta=0.45, \nu=0.1, \Lambda=0$ and $\Gamma_{0}=0.4$. Regions of almost time-periodic flow (sharp lines) separate regions of chaotic dynamics.
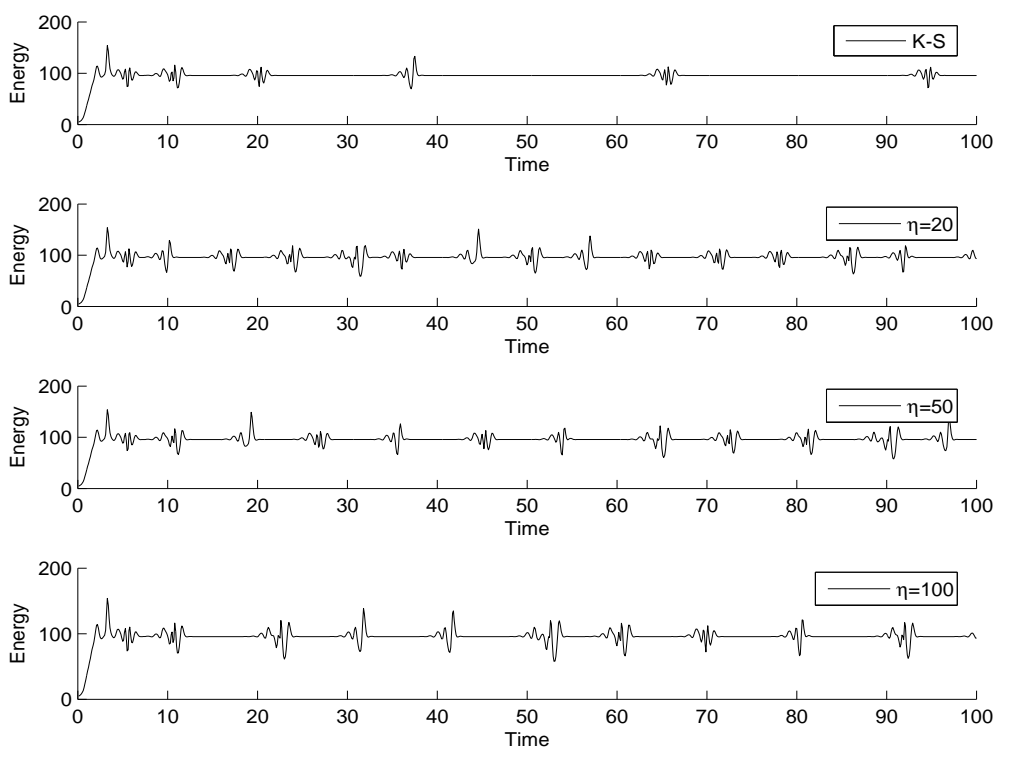

Figure 13. Effect of $\eta$ (values shown) on the dynamics; $\nu=0.1, \Lambda=0, \Gamma_{0}=0.4$. The top panel is the clean case (KS equation). The initial conditions are $H_{0}(z)=\cos z+(1 / 2) \sin 3 z, G_{0}=0.4$.

bifurcation to another time-periodic travelling waves window. This in turn looses stability to tetra-modal steady-state travelling waves, and the scenario is repeated with windows shrinking in size as $\nu$ decreases. We managed to compute octa-modal and decahexa-modal steady-state travelling waves (these are periodic in space with periods $2 \pi / 8$ and $2 \pi / 16$, respectively; equivalently the only non-zero Fourier modes in the spectrum of the solutions are multiples of 8 and 16 , respectively).

In Table 1 we provide details of the windows for $0.2 \leq \nu \leq 0.0008$. In the time periodic windows $B_{2}$ - $B_{5}$ the energy $E(t)$ mostly has two maxima and two minima. The periods of oscillation decrease from roughly 0.48 in window $B_{2}$, to 0.15 in window $B_{3}$, to 0.045 in window $B_{4}$ and finally approximately 0.015 in window $B_{5}$ (the numbers given are representative values - the period varies smoothly with $\nu$ in a given sub-window). The decrease in the period along with the value of $\nu$ impose computational restrictions on time-steps and the number of modes. For example, in window $B_{5}$ we used a time-step $\Delta t=10^{-3}$ and 2048 modes. 


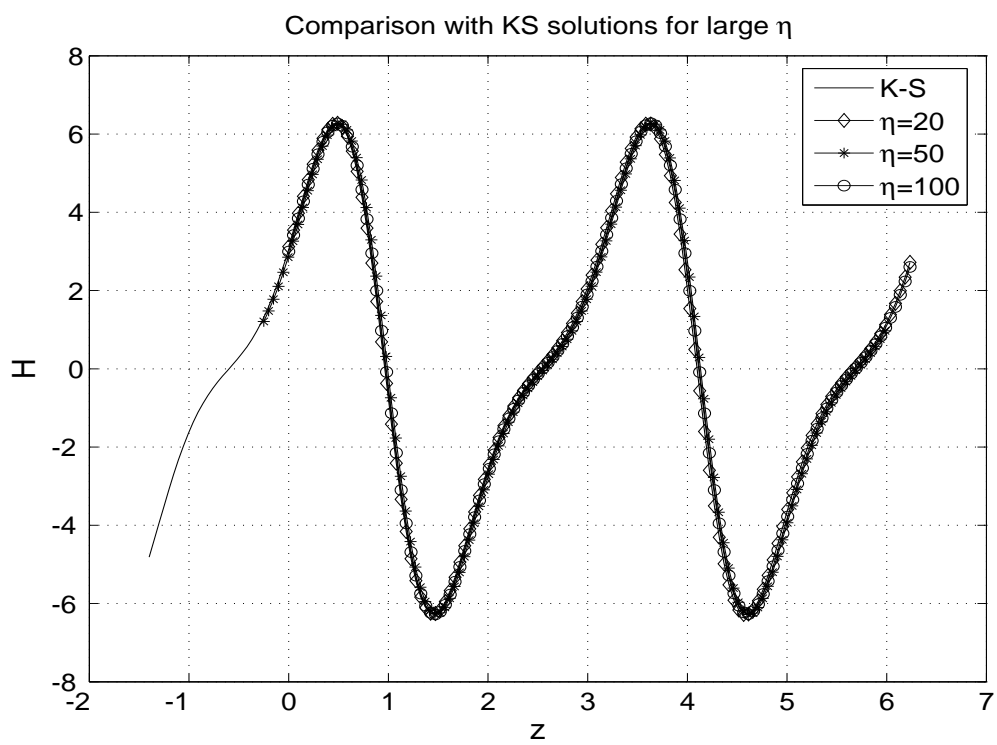

Figure 14. Comparison of the profiles $H$ taken from the constant energy intervals of figure 13. Solid line, KS equation; diamonds, $\eta=20$; asterisks, $\eta=50$; open circles, $\eta=100$. The profiles have been shifted to align horizontally.

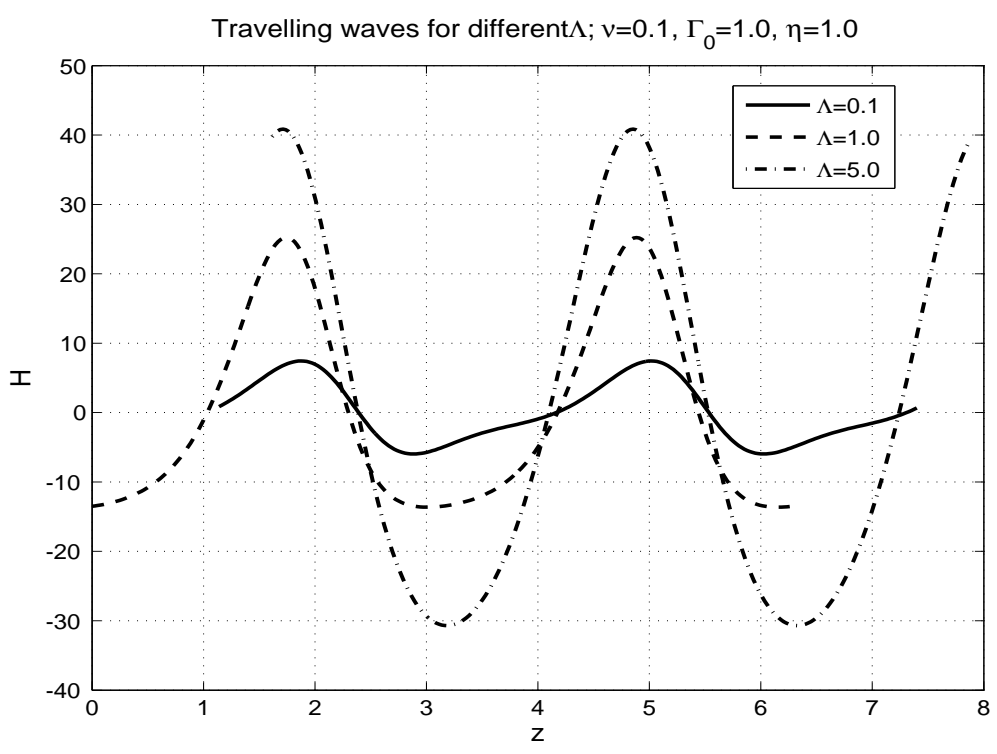

FIgURE 15. Travelling wave profiles at increasing $\Lambda$ (values shown in the plot); $\nu=0.1, \Gamma_{0}=1.0, \eta=1.0$.

In figure 7 we show representative solutions at a fixed large time (so that transients are not present and the waves are travelling). Along with the scaled interfacial shape $H$ and surfactant concentration $\Gamma$, we superimpose the axial velocity $W_{2}(1, z, t)$ (see equation (3.24)). A common feature of the results in figure 7 (and also of travelling waves in windows $A_{4}, A_{8}$ and $A_{16}$ shown later) is that the surfactant concentration has local maxima just ahead of an interfacial local maximum. This can be explained by considering the variation of the local axial velocity $W_{2}$ which is solely responsible for the non-uniform convection of surfactants along the interface, since we are in a travelling frame of reference. In all cases, there is a zero of $W_{2}$ at a position $z=z_{s}$, ahead of an interfacial maximum, such that the local flow for $z>z_{s}$ is negative while for $z<z_{s}$ it is positive. This in turn causes surfactant to accumulate in the vicinity of $z_{s}$ resulting in a surfactant maximum there. The situation at interfacial minima is similar in that there is again a stagnation point ahead of the minimum but the flow switches direction as compared to the previous case and so sweeps the 
Window label Window range in $\nu$ Description of the attractors

\begin{tabular}{ccc}
\hline & & \\
$A$ & $\nu \geq 1.14$ & Trivial solution \\
$A_{1}$ & $0.2 \leq \nu<1.47$ & Fully modal travelling \\
$B_{1}$ & $0.099 \leq \nu \leq 0.19$ & Time-periodic travelling \\
$A_{2}$ & $0.055 \leq \nu \leq 0.098$ & Bi-modal travelling \\
$B_{2}$ & $0.041 \leq \nu \leq 0.054$ & Time-periodic travelling \\
$A_{4}$ & $0.014 \leq \nu \leq 0.04$ & Tetra-modal travelling \\
$B_{3}$ & $0.011 \leq \nu \leq 0.013$ & Time-periodic travelling \\
$A_{8}$ & $0.00375 \leq \nu \leq 0.01$ & Octa-modal travelling \\
$B_{4}$ & $0.00275 \leq \nu \leq 0.0035$ & Time-periodic travelling \\
$A_{16}$ & $0.0009 \leq \nu \leq 0.0025$ & Decahexa-modal travelling \\
$B_{5}$ & $0.000705 \leq \nu \leq 0.0008$ & Time-periodic travelling \\
& & \\
\hline
\end{tabular}

TABLE 1. Solution branches as $\nu$ decreases; $\Lambda=0, \eta=1, \Gamma_{0}=1 . A_{j}$ are windows of steady-state travelling waves of period $2 \pi / j ; B_{1}, \ldots, B_{5}$ are time-periodic windows at successively smaller values of $\nu$.

interface relatively clean of surfactant. This explains the clean regions ahead of an interfacial minimum as seen in the four panels of figure 7. Representative waves from the higher modal travelling wave solutions presented in Table 1 are given in figures 8 and 9. Figure 8 shows the wave shape, surfactant concentration and axial velocity for the two representative values $\nu=0.034,0.021$ which belong to the tetra-modal window $A_{4}$ of Table 1 . The surfactant distribution mechanism described above is also present here but operating on the shorter basic period $\pi / 4$. In figure 9 we provide representative travelling wave solutions at the values $\nu=0.009$ and $\nu=(0.009 / 4)=0.00225$ from the octa-modal and decahexa-modal windows $A_{8}$ and $A_{16}$, respectively. It is observed from figure 9 that as $\nu$ is reduced by a factor of 4 , the amplitude of the decahexamodal waves (bottom panel) increases by a factor of 2 . We note that the wave-speed also increases by a factor of 2. The amplitude of the surfactant concentration is almost unchanged as would be expected by the integral conservation property. These observations reflect an asymptotic scaling property of the equations as we explain next.

Consider a travelling wave solution of equations (5.5) and (5.6) for $\Lambda=0$ denoted by $H(z-c t ; \nu, \eta)$ and $\Gamma(z-c t ; \nu, \eta)$. For a fixed positive integer $\kappa$, substitution of $(5.5)$

$$
H(z, t)=\kappa \bar{H}(y ; \bar{\nu}, \eta), \quad \Gamma(z, t)=\bar{\Gamma}(y ; \bar{\nu}, \eta)
$$

into (5.4) and (5.5), with

$$
\bar{\nu}=\kappa^{2} \nu, \quad y=\kappa(z-c t)
$$

shows that $\bar{H}$ and $\bar{\Gamma}$ satisfy the equations

$$
\begin{aligned}
-c \bar{H}_{y}+\bar{H} \bar{H}_{y}+\bar{H}_{y y}+\bar{\nu} \bar{H}_{y y y y}+\frac{1}{\kappa} \bar{\Gamma}_{y y}=0, \quad 0 \leq y<2 \pi, \\
-c \bar{\Gamma}_{y}+(\bar{H} \bar{\Gamma})_{y}=\eta \bar{\Gamma}_{y y} .
\end{aligned}
$$

Except for the term $\frac{1}{\kappa} \bar{\Gamma}_{y y}$, equations (6.4)-(6.5) have the same form as form as the travelling wave equations for $H(z-c t ; \nu, \eta)$ and $\Gamma(z-c t ; \nu, \eta)$. This suggests the following construction to obtain travelling waves at a decreasing sequence of $\nu$. Starting with $\kappa=1$ and a known value of $\nu$ which supports travelling waves (for example from window $A_{1}$ of Table 1 ), we use the near scale invariance to obtain approximate travelling wave solutions for a decreasing sequence of $\nu=\bar{\nu} / \kappa^{2}$. These approximate solutions are used in the initial value problem (cr)-(cr) to verify that exact, stable travelling wave solutions are asymptotically close to the approximate solutions. The numerical results of figure 9 confirm this observation. The figure shows $H, \Gamma$ 
for values of $\nu$ which correspond to octa-modal and hecadexa-modal waves, i.e. $\kappa=8$ and 16 with respect to a value of $\bar{\nu}$ in window $A_{1}$. In going from $\nu=0.009$ to $\nu=0.00225$, a decrease by a factor of 4 , we apply the transformation (6.2) with $\kappa=2$. Therefore, the profile in the bottom panel of figure 9 is obtained from that in the top panel by multiplying the latter by 2 and horizontally re-scaling it by $1 / 2$. In figure 10 , we superimpose a re-scaled $\nu=0.009$ profile over the profile at $\nu=0.00225$, using open circles for the former The corresponding re-scaling for $\Gamma$ is given in figure 11. The agreement is excellent showing that the approximate travelling waves obtained by re-scaling are very close to the exact travelling wave solutions even for moderate values of $\kappa$. We use this property to find windows of travelling wave solutions at decreasing values of $\nu$.

In the next set of numerical experiments we evaluate the effect of the diffusion coefficient $\eta$ on the solutions for fixed values of $\nu=0.1$ and $\Gamma_{0}=0.4$ with initial condition $H_{0}(z)=\cos z+(1 / 2) \sin 3 z, G_{0}(z)=0.4$. The value $\nu=0.1$ is chosen because it produces, in the absence of surfactants, chaotic homoclinic bursts (see Smyrlis \& Papageorgiou(1996a)), which is expected to be reproduced at large $\eta$ since the system would then behave as if it were clean. The attractors are well defined at relatively small and large values of $\eta$. At intermediate values, however, it is not clear (even after computations of several thousand time-units) if a well-defined final state emerges. For example, for values of $\eta \in[0.001,0.2]$ the flow is time-periodic with four well defined maxima and four minima in the signal of $E(t)$. Time periodicity is lost for higher values of $\eta$ and in particular for $\eta \in[0.35,0.6]$, our numerical solutions produce complex dynamics which include sequences of almost time-periodic intervals with chaotic dynamics in between.

A representative example is given in figure 12 for $\eta=0.45$. The figure depicts the evolution of the maxima of $E(t)$ over a time interval $2 \times 10^{4}$. The picture is produced by plotting a dot every time a maximum of $E(t)$ is detected and it is seen that the dynamics has not reached a final state (and probably does not do so) but is exhibiting complex behavior. For example, in regions where there is a cloud of dots (the time intervals $3 \times 10^{3}-9 \times 10^{3}$ and $1.3 \times 10^{4}-1.7 \times 10^{4}$, approximately) the flow is chaotic, as quantified by return maps. Just before the second chaotic window mentioned above, the flow is almost periodic with six maxima (and six minima) - exact periodicity would be seen as points lying on six parallel horizontal lines. In addition, it appears that the route to chaos is through a period doubling cascade as seen by the branching of the periodic solutions near $t \approx 1.2 \times 10^{4}$. As the flow evolves the chaotic windows are interchanged with almost periodic windows and the pattern persists.

As $\eta$ is increased above 0.6, the large time behavior is mostly chaotic. In order to make a direct comparison with solutions of the KS equation, we consider the effect of increasing $\eta$. For values of $\eta$ larger than about 10, the dynamics is dominated by chaotic homoclinic bursts, as are the dynamics of the KS solutions also included in figure 13. In the homoclinic bursts, the $L^{2}$-norm of $H$ undergoes rapid chaotic oscillations which are separated by equal constant states (the dynamics are homoclinic). The separation between chaotic bursts (equivalently the duration of the constant energy states) becomes longer as $\eta$ is increased, with the clean case (KS equation) having the longest separations. In the time intervals where $E(t)$ is constant, the flow is a bimodal steady-state travelling wave. A direct comparison of the computed interfacial profiles taken from any of the constant $E$ intervals is made in figure 14. The behavior of the dynamics tending to that of the Kuramoto-Sivashinsky equation as $\eta$ becomes large, is expected to hold at other values of $\nu$ also. It is also noteworthy that $\eta$ does not need to be very large for agreement with KS dynamics to hold.

\subsection{The case of unequal viscosities, $\Lambda \neq 0$}

Next we turn to the numerical solutions of the canonical evolution equations (5.6) and (5.5) when $\Lambda \neq 0$. Due to the increased number of parameters (there are four now), we have not attempted to construct a complete picture of the solution space. Instead, we concentrate on the effect of $\Lambda$ on the dynamics.

Through numerical experiments we have established that the effect of $\Lambda$ on travelling wave solutions (for example solutions supported in window $A_{1}$ of Table 1 ) is to increase the wave speed and the amplitudes of the solutions (equivalently the $L^{2}$-norm).

Of more interest is the effect of $\Lambda$ on solution branches which are not travelling waves but which evolve dynamically, for example time-periodic. It has been established for the clean case that the dispersive effects introduced by the viscosity stratification act to regularize dynamic complexity into pulses of periodic travelling waves with increasing amplitudes - see Papageorgiou et al. (1990). In what follows we compute such phenomena for the surfactant-laden interface. We choose $\nu=0.1, \eta=1$ and $\Gamma_{0}=1$, which for $\Lambda=0$ corre- 
Window range in $\Lambda$
Description of the attractors

$$
\begin{gathered}
0 \leq \Lambda \leq 0.0011 \\
0.0012 \leq \Lambda \leq 0.0017 \\
0.002 \leq \Lambda \leq 0.0021 \\
0.0023 \leq \Lambda \leq 0.0093 \\
0.013 \leq \Lambda \leq 0.02 \\
0.021 \leq \Lambda \leq 0.034 \\
0.035 \leq \Lambda \leq 0.0401 \\
0.0402 \leq \Lambda \leq 0.0404 \\
\Lambda=0.0405 \\
0.0406 \leq \Lambda \leq 0.0409 \\
0.041 \leq \Lambda \leq 0.042 \\
0.043 \leq \Lambda \leq 0.072 \\
0.074 \leq \Lambda \leq 5.0
\end{gathered}
$$

Time periodic TP5

Time periodic TP10

Time periodic TP20

Chaotic oscillations

Time periodic TP1, TP2, TP4

Chaotic oscillations (with small embedded TP5, TP25 windows)

Time periodic TP1, TP5, TP8, TP24

Chaotic oscillations

Time periodic TP8

Time periodic TP4

Time periodic TP2

Time periodic TP1

Bimodal steady-state travelling

TABLE 2. Solution branches as $\Lambda$ increases; $\nu=0.1, \Gamma_{0}=1.0, \eta=1.0$

sponds to a solution is in the lower part of sub-window $B_{1}$ of Table 1 and is characterized by five maxima and five minima (labeled by TP5 where the numeral after TP denotes the number of maxima and minima) in the signal of $E(t)$. The period of oscillation is $T \approx 3.053$. Over a hundred numerical experiments have been performed to compile results for non-zero $\Lambda$ which are shown in Table 2 .

The overall trend, as $\Lambda$ increases from zero, is to enter a chaotic regime through what appears to be a subharmonic cascade, and eventually to obtain solutions attracted to bimodal steady-state travelling waves through a reverse subharmonic cascade. More precisely, the time periodic TP5 branch of solutions that starts at $\Lambda=0$, period doubles to a TP10 branch at a value of $\Lambda \in(0.0011,0.0012)$. The TP10 branch in turn period doubles to a TP20 branch of solutions at a value of $\Lambda \in(0.0017,0.002)$, and chaotic oscillations are established at a value of $\Lambda$ between 0.0021 and 0.0023 . Using the first three windows of Table 2 we find that the ratio of successive window sizes to be 2.2 and 5.0, although these values can be sharpened significantly with additional computations. According to the Feigenbaum scenario, the sizes of successive periodic windows (as an accumulation point beyond which chaos emerges), decrease geometrically with the asymptotic ratio equal to 4.6692016 ... (see Feigenbaum (1978), Feigenbaum(1983), and Collet and Eckmann (1980). ) Our numerical results are consistent with the universal theory (the analogous computation for the Kuramoto-Sivashinsky equation produced three-digit accuracy, see Smyrlis and Papageorgiou 1991).

The chaotic window $0.0023 \leq \Lambda \leq 0.0093$ can be characterized as Feigenbaum chaos, being a result of subharmonic cascade. At values of $\Lambda>0.0093$ the dynamics is governed by an interplay between different attractors and their basins of attraction (note, that computationally we are accessing the most attracting solutions since we solve initial value problems). As can be seen from Table 2, the Feigenbaum chaos window gives way to time-periodic TP1 solutions at $\Lambda \approx 0.013$. As $\Lambda$ is increased further, subharmonic bifurcations take place to produce two period doubled windows TP2 and TP4. Even though the results are not extensive, we estimate the ratio of the lengths of the windows TP2 to TP4, to be approximately equal to 4, again in general agreement with the Feigenbaum route to chaos. This chaotic window persists with time periodic solutions of different types in between (see Table 6.2). The last window of chaotic oscillations is in the small interval $0.0402 \leq \Lambda \leq 0.0404$. Further increase of $\Lambda$ produces the time periodic solutions TP8, TP4, TP2 and TP1 shown in the Table. The largest $\Lambda$ that produces a time periodic solution (TP1) in our numerical experiments is $\Lambda=0.072$. At larger values the solutions get attracted to bimodal steady-state travelling waves. It is interesting to start with the bimodal travelling waves and to decrease $\Lambda$. Using the results of Table 6.2, we can see that the small chaotic window in the interval $0.0402 \leq \Lambda \leq 0.0404$, appears to be the 
result of a period doubling route to chaos. The computed window lengths for TP4, TP2 and TP1 solutions are $0.0003,0.001$ and 0.03 , yielding an estimate for the universal constant of approximately 3.3 - as mentioned earlier this can be improved significantly but this is not our main interest here. We conclude, therefore, that as $\Lambda$ increases from zero a window of chaotic oscillations emerges through a Feigenbaum period doubling scenario.

On further increase of $\Lambda$, the chaotic attractor regularizes to time periodic solutions which appear to follow a reverse Feigenbaum cascade (as $\Lambda$ increases) to bimodal steady-state travelling waves. Figure 15 presents representative travelling waves for the values $\Lambda=0.1,1.0,5.0$. The results show that the travelling wave amplitudes and speeds increase monotonically with increasing $\Lambda$.

Finally, we discuss briefly solutions for $\Lambda<0$ starting with the time periodic TP5 solutions corresponding to $\Lambda=0, \nu=0.1, \eta=1$ and $\Gamma_{0}=1$, as above. The behavior follows the dynamics for $\Lambda>0$ (see Table 2) but the windows of the different attractors are not delineated as well. For example, we find time periodic TP5 solutions for $-0.0025 \leq \Lambda \leq 0$ and bimodal travelling waves for $\Lambda \leq-0.004$.

The general features of the monotonically increasing amplitudes and of the travelling waves as $|\Lambda|$ is increased have also been observed. A notable difference is that for $\Lambda<0$ the travelling waves have positive wave speeds. Our results indicate that the speeds of the travelling waves are asymptotically the same at large $|\Lambda|$, the difference being the sign of the wave-speed. A heuristic argument that confirms this follows from equation (5.6) written in a Galilean frame of speed $c$, say,

$$
-c H_{z}+H H_{z}+2 \frac{\Lambda}{\sqrt{\nu}} H_{z}-\frac{\Lambda \sqrt{\nu}}{6} H_{z z z}+H_{z z}+\nu H_{z z z z}+\Gamma_{z z}=0 .
$$

When $\Lambda \gg 1$, the Kuramoto-Sivashinsky terms $H_{z z}+\nu H_{z z z z}$ and the surfactant term $\Gamma_{z z}$ are of lower order and the equation appears to represent travelling waves of the Kortweg de-Vries equation, to leading order (the scaling $H \sim \Lambda$ is implied). The following symmetry of travelling waves of the $\mathrm{KdV}$

$$
H \rightarrow-H, \quad \Lambda \rightarrow-\Lambda, \quad c \rightarrow-c,
$$

is consistent with the numerical observations above. We emphasize that this argument is heuristic and a more complete computational and asymptotic study for large $|\Lambda|$ is necessary.

\section{Conclusions}

The problem of the nonlinear stability of core-annular flows with surfactant-laden interfaces has been addressed analytically and numerically. We have developed an asymptotic theory, in the thin annulus limit, that captures the first stages of nonlinear spatiotemporal dynamics. The asymptotic development is based on identifying canonical nonlinear regimes, in which consistent asymptotic models are possible if the initial surfactant concentration is chosen appropriately (scaling regimes outside the ones characterized here, lead to linear dynamics as studied in detail by Wei \& Rumschitzki 2005). In particular, the analysis holds in the dilute surfactant concentration limit and for high Marangoni numbers.

Extensive numerical experiments have been carried out to classify the dynamics over a wide range parameters. In the absence of viscosity differences we find that as $\nu$ decreases, or equivalently the size of the system increases, the solutions are mostly steady-state travelling waves with successively higher modal characteristics - that is, the basic spatial period decreases as $\nu$ is decreased. Windows supporting steady-state travelling waves are separated by narrow windows (in $\nu$ ) which produce time-periodic travelling waves; these are solutions which reproduce the original profile after one time period of oscillation, but shifted horizontally. A summary of typical results for the case $\Lambda=0, \eta=1, \Gamma_{0}=1$, is given in Table 1 . We see that successive steady-state travelling wave windows have their spatial period successively halved. We have also established that these solutions are asymptotically self-similar as $\nu$ decreases, and have shown how a $2 \pi$ spatially periodic wave generates waves of period $2 \pi / N$ (where $N$ is an integer) from higher windows - see figures 10,11 for example.

The effects of varying the dimensionless surfactant diffusion coefficient $\eta$ has also been studied in the absence of viscosity differencers. Intuitively, we expect that for large $\eta$, strong diffusion will make the surfactant distribution uniform and thus remove Marangoni effects, so that the dynamics are similar to the clean case. Our results show that if $\eta$ is small the solution is time-periodic with four maxima and minima in the time 
series of its energy, for example. For larger $\eta$ complex dynamics develop with intervals of time-periodic solutions separated by chaotic attractors - see figure 13 for example. The expected agreement to the dynamics of the clean case occurs when $\eta$ is larger than about 20 as is shown in figures 13 and 14 .

When viscosity differences are present $(\Lambda \neq 0)$, the nonlinear evolution equations contain non-local terms which can be purely dispersive. It has been shown by Papageorgiou et al. (1990) and Smyrlis \& Papageorgiou (1998), that such dispersive effects act to suppress chaotic dynamics, instead giving travelling wave pulses. We have studied such phenomena in the presence of surfactant and the results of a large number of numerical experiments are given in section 6.2 and Table 2. It is found that as $\Lambda$ increases through positive values, a period doubling route to chaos takes place with chaotic oscillations setting in by $\Lambda=0.0023$. Further increase of $\Lambda$ supports dynamics which are chaotic with regions of time-periodic solutions with different characteristics. The largest computed $\Lambda$ values that produce chaotic dynamics are in the range $0.0402 \leq \Lambda \leq 0.0404$. At $\Lambda=0.0405$ the solution is time-periodic with eight maxima and eight minima in the energy norm time series. A further increase in $\Lambda$ produces a reverse Feigenbaum cascade, with a strongly attracting bimodal steady-state travelling wave window for $\Lambda \geq 0.074$. Representative profiles in the travelling-wave window are depicted in figure 15 for $\Lambda=0.1,1.0,5.0$. The amplitudes of the waves are seen to increase with $\Lambda$ and so do the wave speeds. When $\Lambda<0$ (i.e. $m>1$ implying that the film is more viscous than the core), we find that the direction of the travelling waves changes and that the speeds are asymptotically equal and opposite at large $|\Lambda|$. A heuristic explanation of this is also presented and is based on the fact that the leading order system at large $\Lambda$ is similar to the Kortweg de-Vries equation.

As a representative physical example we consider oil-water displacement in porous media. Following Wei $\&$ Rumschitzki (2005) we consider an oil film with viscosity $\mu_{2}=10 \mathrm{cP}$ surrounding a water core with $\mu_{1}=1$ cP giving $m=10$. Other representative values are $\sigma_{0}=10 \mathrm{dyn} / \mathrm{cm}$, a tube diameter $R_{2}=100 \mu m$, a slug velocity of $1 \mathrm{~cm} / \mathrm{s}$ or less, and an insoluble surfactant concentration in the range $10^{-12}$ to $10^{-10} \mathrm{~mol} \mathrm{~cm}^{-2}$. Using a typical value for $\Gamma_{\infty}=10^{-9}-10^{-10} \mathrm{~mol} \mathrm{~cm}^{-2}$ (Chang \& Franses 1995) gives an estimate for our dimensionless initial surfactant concentration $\Gamma_{0}=10^{-3}-1$ as required by (3.13) and (3.20)-(3.22). Other dimensionless parameters are $R_{e 1}$ is 1 or less, $C_{a} \sim 10^{-2}$ and $M_{a}$ is in the range 2.5 to 250 . Since $C_{a}$ in the example is small, for coupling between film and core the theory demands very small values of $\epsilon$ (see comment following equation (3.9)). It follows that for values of $\epsilon$ greater than about $10^{-2}$ the present theory is applicable by taking $\Lambda=0$ (i.e. no coupling between core and film). Our numerical computations indicate that steady-state travelling wave solutions can exist in the presence of surfactants.

A second example arises in lubricated pipelining and the experiments of Aul \& Olbricht (1990). The experiments are performed in glass capillary tubes of circular cross-section with outer radius $R_{2}=27 \mu \mathrm{m}$. The annular fluids used are UCON oils with a range of different viscosities $\mu_{2}=1.70 P, 0.79 P, 0.19 P$, and the core fluid is water with viscosity $\mu_{1}=10^{-2} P$ (the fluids are chosen to be of almost equal densities). Several experiments are reported (for example Table 2 of Aul \& Olbricht 1990), which have film thicknesses $R_{2}-R_{1}=1.8 \mu m, 1.6 \mu m, 1.0 \mu m$, respectively, with corresponding viscosity ratios $m=173,80,19$ and Capillary numbers $C_{a}=0.022,0.010,0.0024$. The corresponding values of our dimensionless parameter $\epsilon=$ $\frac{R_{2}-R_{1}}{R_{1}}$ are $0.0714,0.063$ and 0.0385 . The surface tension coefficient is $\sigma_{0}=3.5 d y n e / \mathrm{cm}$ and the Reynolds numbers based on the core fluid (as in the present study) for the experiments were small and always smaller than 0.019; the parameter $J$ introduced in (2.20) is of order one and approximately equal to 94.5. Aul \& Olbricht (1990) observed instability for all systems and the eventual evolution of the system to a nonlinear traveling wave train of axisymmetric lobes or collars which are spaced periodically along the tube (the reader is referred to figure 4 of Aul \& Olbricht 1990). As in the previous example the values of $C_{a}$ are smaller than the canonical scaling $C_{a}=m \epsilon$ (see (3.9)) and this implies that the film and core dynamics decouple and the appropriate mathematical model developed here has $\Lambda=0$. The measured wavelengths were approximately $225 \mu \mathrm{m}$ which corresponds to a dimensionless wavelength of approximately $L=8.9$. This can be used to estimate the value $\nu=\frac{\pi^{2}}{L^{2}} \approx 0.125$ to be used in the evolution equations (5.4)-(5.5), along with $\Lambda=0$. In the absence of surfactants it has been shown by Smyrlis \& Papageorgiou (1996) that for $\nu=0.125$ the dynamics of the Kuramoto-Sivashinsky model is attracted to a chaotic temporal state. The numerical results of the present study show that surfactants on the whole tend to organize such dynamics into traveling wave pulses. It is not possible to quantify the effect of surfactants on the experiments of Aul \& Olbricht (1990), but it is reasonable to assume that they were present at least as trace impurities, a limit which is covered by our 
theory. We also emphasize that the pinching of the core fluid reported by Aul \& Olbricht (1990) is a fully nonlinear phenomenon and beyond the reach of our weakly nonlinear theory.

SAK wishes to thank the Consejo de Investigación of Universidad de Oriente through grant number CI2-010301-1277/06. The work of DTP was supported in part by the National Science Foundation Grant number DMS-0072228. The work MS was supported by the National Science Foundation Grant number DMS-0354560. In addition we acknowledge the NSF grant MRI DMS-0420590 for computing resources.

\section{REFERENCES}

Aul, R. W. \& Olbricht, W. L. 1990 Stability of thin annular film in pressure-driven, low-Reynolds-number flow through a capillary. Journal of Fluid Mechanics 215, 585-599.

Blyth, M.G., Hall, P. \& Papageorgiou, D. T. 2003 Chaotic flows in pulsating cylindrical tubes: A class of exact solutions of the navier-stokes equations. Journal of Fluid Mechanics 481, 187-213.

Blyth, M. G., Luo, H. \& PozRikidis, C. 2006 Stability of axisymmetric core-annular flow in the presennce of an insoluble surfactant. Journal of Fluid Mechanics 548, 207-235.

Blyth, M. G. \& PozRikidis, C. 2004 Effect of surfactants on the stability of two-layer channel flow. Journal of Fluid Mechanics 505, 59-86.

Chang, H.-C. \& Franses, E.I. 1995 Adsorption dynamics of surfactants at the air/water interface: a critical review of mathematical models, data, and mechanisms. Colloids and Surfaces A: Phy. Eng. Aspects 100, 1-45.

Charru, F. \& Hinch, E. J. 2000 "phase diagram" of interfacial instabilities in two-layer couette flow and mechanism of the long-wave instability. Journal of Fluid Mechanics 414, 195-223.

Collet, P. \& Eckmann, J.-P. 1980 Iterated maps on the interval as dynamical systems. Boston, U.S.A.: Birkhauser.

Craster, R. V., Matar, O. K. \& Papageorgiou, D. T. 2002 Pinchoff and satellite formation in surfactant covered viscous threads. Physics of Fluids 14, 1364-1376.

Feigenbaum, M. J. 1978 Quantitive universality for a class of nonlinear transformations. Journal of Statistical Physics 19, 25-52.

Feigenbaum, M. J. 1983 Universal behavior in non-linear systems. Physica D 7, 16-39.

Frenkel, A. L., Babchin, A. J., Levich, B. G., Shlang, T. \& Sivashinsky, G. I. 1987 Annular flows can keep unstable films from breakup: Nonlinear saturation of capillary instability. J. Colloid Interface Science 115, 225.

Frenkel, A. L. \& Halpern, D. 2002 Stokes-flow instability due to interfacial surfactant. Phys. Fluids 14, L45-L48.

Georgiou, E., Maldarelli, C., Papageorgiou, D. T. \& Rumschitzki, D. S. 1992 An asymptotic theory for the linear stability of core-annular flow in the thin annular limit. Journal of Fluid Mechanics 243, 653-677.

Goren, S. L. 1962 The instability of annular thread of fluid. Journal of Fluid Mechanics 12, 309-319.

Halpern, D. \& Grotberg, J. B. 1993 Surfactant effects on fluid-elastic instabilities of liquid-lined flexible tubes: A model of airway closure. Journal of Biomechanical Engineering 115, 271-277.

HAMmond, P. S. 1983 Nonlinear adjustment of a thin annular film of viscous fluid surrounding a thread of another within a circular cylinder pipe. Journal of fluid Mechanics 137, 363-384.

Hansen, S., Peters, G. W. M. \& MeiJer, H. E. H. 1999 The effect of surfactant on the stability of a fluid filament embedded in a viscous fluid. Journal of fluid Mechanics 382, 331-349.

Hickox, C. E. 1971 Stability due to viscosity and density stratification in axisymmetric pipe flow. Physics of Fluids 14, 251 .

Joseph, Daniel D. \& Renardy, Yuriko Y. 1993 Fundamentals of Two-Fluid Dynamics. Part II: Lubricated Transport, Drops and Miscible Liquids. New York: Springer-Verlag.

Kas-Danouche, S. 2002 Nonlinear interfacial stability in core-annular films flows in the presence of surfactants. Newark, U.S.A.: New Jersey Institute of Technology.

Kerchman, V. 1995 Strongly nonlinear interfacial dynamics in core-annular flows. Journal of Fluid Mechanics 290, $131-166$.

Kwak, S., Fyrillas, M. M. \& Pozrikidis, C. 2001 Effect of surfactants on the instability of a liquid thread Part 2. Extensional flow. Int. J. Multiphase Flow pp. 1-22.

Kwak, S. \& PozRikidis, C. 2001 Effect of surfactants on the instability of a liquid thread or annular layer. Part 1. Quiescent fluids. Int. J. Multiphase Flow 27, 1-37.

Newhouse, L. A. \& PozRikidis, C. 1992 The capillary instability of annular layers and liquid threads. Journal of Fluid Mechanics 242, 193-209.

Otis, D. R., Jr., Johnson, M., Pedley, T. J. \& Kamm, R. D. 1990 The effect of surfactant on liquid film stability in the peripheral airways. Advances in Bioengineering 17, 55-57.

Otis, D. R., JR., Johnson, M., Pedley, T. J. \& Kamm, R. D. 1993 Role of pulmonary surfactant airway closure: a computational study. Journal of Applied Physiology 75, 1323-1333.

Papageorgiou, D. T., Maldarelli, C. \& Rumschitzki, D. S. 1990 Nonlinear interfacial stability of core-annular film flows. Physics of Fluids A 2 (3), 340-352. 
Papageorgiou, D. T. \& Smyrlis, Y. S. 1991 The route to chaos for the Kuramoto-Sivashinsky equation. Teoret. Comput. Fluid Dynamics 3, 15-42.

PARK, C. W. \& Homsy, G. M. 1984 Two-phase displacement in hele-shaw cells: theory. Journal of Fluid Mechanics 141, 257-287.

Preziosi, L., Chen, K. \& Joseph, D. D. 1989 Lubricated pipelining: Stability of core-annular flow. Journal of Fluid Mechanics 201, 323-356.

Slattery, J. C. 1974 Interfacial effects in the entrapment and displacement of residual oil. AIChE J. 20, $1145-1154$.

Smyrlis, Y. S. \& Papageorgiou, D. T. 1991 Predicting chaos for infinite dimensional dynamical systems: The Kuramoto-Sivashinsky equation, a case of study. Proc. Natl. Acad. Sci. USA 88, 11129-11132.

Smyrlis, Y. S. \& Papageorgiou, D. T. 1996 a Computational study of chaotic and ordered solutions of the Kuramoto-Sivashinsky equation. Tech. Rep. ICASE Report No. 96-12. NASA Contract No. NAS1-19480. Institute for Computer Applications in Science and Engineering. NASA Langley Research Center, Hampton, Virginia.

Smyrlis, Y. S. \& Papageorgiou, D. T. $1996 b$ Computational study of chaotic and ordered solutions of the kuramoto-sivashinsky equation. Tech. Rep.. NASA Langley Research Center, Hampton, Virginia.

Smyrlis, Y. S. \& Papageorgiou, D. T. 1998 The effects of generalized dispersion on dissipative dynamical systems. Appl. Math. Lett. 11 (6), 93-99.

Timmermans, M. L. E. \& Lister, J. R. 2002 The effect of surfactant on the stability of a liquid thread. Journal of Fluid Mechanics 459, 289-306.

TомотіKA, S. 1935 On the instability of a cylindrical thread of a viscous liquid surrounded by another viscous liquid. Proc. R. Soc. Lond. A 150, 322-337.

Wei, H. H. \& Rumschitzki, D. S. 2005 The effects of insoluble surfactants on the linear stability of core-annular flow. Journal of Fluid Mechanics 541, 115-142.

Wong, H., Rumschitzki, D. \& Maldarelli, C. 1996 On the surfactant mass balance at a deforming fluid interface. Physics of Fluids 8 (11), 3203-3204.

YIH, C. S. 1967 Instability due to viscosity stratification. Journal of Fluid Mechanics 27, 337-352. 